\title{
Rotational dependence of turbulent transport coefficients in global convective dynamo simulations of solar-like stars
}

\author{
J. Warnecke ${ }^{1}$ and M. J. Käpylä ${ }^{1,2}$ \\ 1 Max-Planck-Institut für Sonnensystemforschung, Justus-von-Liebig-Weg 3, 37077 Göttingen, Germany \\ e-mail: warnecke@mps.mpg.de \\ 2 ReSoLVE Centre of Excellence, Department of Computer Science, Aalto University, PO Box 15400, 00076 Aalto, Finland
}

Received 15 October 2019 / Accepted 20 February 2020

\begin{abstract}
Context. For moderate and slow rotation, the magnetic activity of solar-like stars is observed to strongly depend on rotation, while for rapid rotation, only a very weak or no dependency is detected. These observations do not yet have a solid explanation in terms of dynamo theory.

Aims. We aim to find such an explanation by numerically investigating the rotational dependency of dynamo drivers in solar-like stars, that is, stars that have a convective envelope of similar thickness to that of the Sun.

Methods. We ran semi-global convection simulations of stars with rotation rates from 0 to 30 times the solar value, corresponding to Coriolis numbers, Co, of 0 to 110 . We measured the turbulent transport coefficients contributing to the magnetic field evolution with the help of the test-field method, and compared with the dynamo effect arising from the differential rotation that is self-consistently generated in the models.

Results. The trace of the $\alpha$ tensor increases for moderate rotation rates with $\mathrm{Co}^{0.5}$ and levels off for rapid rotation. This behavior is in agreement with the kinetic $\alpha$ based on the kinetic helicity, if one takes into account the decrease of the convective scale with increasing rotation. The $\alpha$ tensor becomes highly anisotropic for Co $\gtrsim 1$. Furthermore, $\alpha_{r r}$ dominates for moderate rotation $(1<\mathrm{Co}<10)$, and $\alpha_{\phi \phi}$ for rapid rotation $(\mathrm{Co} \gtrsim 10)$. The effective meridional flow, taking into account the turbulent pumping effects, is markedly different from the actual meridional circulation profile. Hence, the turbulent pumping effect is dominating the meridional transport of the magnetic field. Taking all dynamo effects into account, we find three distinct regimes. For slow rotation, the $\alpha$ and Rädler effects are dominating in the presence of anti-solar differential rotation. For moderate rotation, $\alpha$ and $\Omega$ effects are dominant, indicative of $\alpha \Omega$ or $\alpha^{2} \Omega$ dynamos in operation, producing equatorward-migrating dynamo waves with a qualitatively solar-like rotation profile. For rapid rotation, an $\alpha^{2}$ mechanism with an influence from the Rädler effect appears to be the most probable driver of the dynamo. Conclusions. Our study reveals the presence of a large variety of dynamo effects beyond the classical $\alpha \Omega$ mechanism, which need to be investigated further to fully understand the dynamos of solar-like stars. The highly anisotropic $\alpha$ tensor might be the primary reason for the change of axisymmetric to non-axisymmetric dynamo solutions in the moderate rotation regime.
\end{abstract}

Key words. magnetohydrodynamics (MHD) - turbulence - dynamo - Sun: magnetic fields - stars: magnetic field - stars: activity

\section{Introduction}

The magnetic activity of stars shows a strong dependency on rotation, which is most pronounced in their coronal X-ray flux (e.g., Pizzolato et al. 2003; Vidotto et al. 2014; Reiners et al. 2014; Wright \& Drake 2016) and their chromospheric Ca II H\&K emission (e.g., Noyes et al. 1984; Brandenburg et al. 1998; Boro Saikia et al. 2018; Olspert et al. 2018). Higher rotation, usually measured using the Coriolis number, Co, describing the rotational influence on convection, leads to stronger coronal and chromospheric emission. For rapidly rotating stars with Co $\gtrsim 10$ (e.g., Wright \& Drake 2016), the emission becomes independent of rotation, which is often referred to as the "saturated" regime. This terminology is somewhat misleading, as it may be confused with the nonlinear saturation of the dynamo; all the dynamos in these stars are indeed expected to be saturated dynamos. The coronal and chromospheric emission can be linked to the surface magnetic field (e.g., Pevtsov et al. 2003; Vidotto et al. 2014), and therefore to the underlying dynamo process. Therefore, it is very important to study the dependency of the dynamo process on rotation.
The most important dynamo effect in astrophysical systems is the $\alpha$ effect (Steenbeck et al. 1966), which describes the ability of small-scale velocity with a twist, for example due to rotation in a stellar convection zone, to amplify the magnetic field. Using mean-field theory with the second-order correlation approximation (SOCA) and assuming isotropic homogeneous turbulence, Steenbeck et al. (1966) found that $\alpha$ increases linearly with rotation in the slow rotation regime because the $\alpha$ effect is directly related to the kinetic helicity under these assumptions. An extension of the theory to higher rotation predicts that the $\alpha$ will level off in this regime (e.g., Ruediger \& Kichatinov 1993). Differential rotation, the other important ingredient of a stellar dynamo process, is predicted to depend only weakly on rotation using the models by Kitchatinov \& Rüdiger (1999). In them, turbulent effects generating the differential rotation are parametrized and obtained from mean-field theory; hence the common reference to "mean-field models". The weak rotational dependence of differential rotation is confirmed by observational studies of the surface latitudinal differential rotation (e.g., Reinhold et al. 2013; Lehtinen et al. 2016).

Global convective dynamo simulations have been able to identify three distinctive regimes in terms of rotation. At 
moderate rotation, where the Coriolis number is between three and ten, these simulations produce cyclic dynamo waves propagating towards the equator (e.g., Ghizaru et al. 2010; Käpylä et al. 2012; Augustson et al. 2015; Strugarek et al. 2017; Warnecke 2018), a basic feature of the solar magnetic field evolution. Recently some of these simulations have even reproduced dynamo solutions with multiple modes with shorter and longer periods than the dominant cycle (Beaudoin et al. 2016; Käpylä et al. 2016, 2017). Most of these cyclic dynamo solutions can be explained by an $\alpha \Omega$ dynamo wave following the ParkerYoshimura rule (Parker 1955; Yoshimura 1975) as shown in the studies by Warnecke et al. (2014, 2018), Käpylä et al. (2016, 2017), and Warnecke (2018). In a classical $\alpha \Omega$ dynamo, the poloidal magnetic field is generated by the $\alpha$ effect from the toroidal magnetic field, which is produced from the shear of the differential rotation ( $\Omega$ effect). To excite an equatorwardmigrating wave, such as the one seen in the Sun, the product of $\alpha$ and the radial shear must be negative(positive) in northern(southern) hemisphere. In these simulations, the sign of $\alpha$ is unfavorable for the correct migration direction of the dynamo wave in the bulk of the convection zone. Instead, most of the simulations produce a local minimum of negative shear, which results in the correct migration direction. However, such a feature is not seen in solar observations, although negative shear is present in the very topmost layer of the convection zone, called the near-surface shear layer (Thompson et al. 1996; Barekat et al. 2014). In the work of Duarte et al. (2016), has equatorward migration been seen in thick convection zones. This was found to result from the reversed sign of kinetic helicity, hence the $\alpha$, in the bulk of the convection zone.

At Coriolis numbers around unity and below, the differential rotation profile develops fast poles and a slow equator, which is opposite to the Sun, with its fast equator and slow poles; hence the name, anti-solar differential rotation profile. In this regime, most of the simulations produce irregular in-time dynamo solutions (Karak et al. 2015; Warnecke 2018). However, Viviani et al. (2018, 2019) discovered a cyclic solution in this regime. None of these dynamo solutions can be explained by a pure $\alpha \Omega$ dynamo as in the moderate rotation case. The study of Viviani et al. (2019) revealed that the $\alpha$ effect generating the toroidal magnetic field is comparable to or even larger than the $\Omega$ effect of differential rotation. In the Coriolis number range between the regime described above, we often find a mixture of both dynamo types (e.g., Viviani et al. 2018; Warnecke 2018).

For large Coriolis numbers, mean-field dynamo models predict dynamo solutions with non-axisymmetric large-scale magnetic field which is often associated with a strong anisotropy of the $\boldsymbol{\alpha}$ tensor (e.g., Rädler et al. 1990; Elstner \& Rüdiger 2007; Pipin 2017). However, non-axisymmetric dynamo solutions have also been obtained with an isotropic $\alpha$ (e.g., Moss \& Brandenburg 1995; Moss et al. 1995; Tuominen et al. 2002). Observational studies also indicate strong nonaxisymmetric surface field (e.g., Morin et al. 2010) or photometric spot distribution (Lehtinen et al. 2016) for stars with a high Coriolis number. Global convective dynamo simulations confirm nonaxisymmetric dynamo solutions for moderately and rapidly rotating stars (Käpylä et al. 2013; Cole et al. 2014; Viviani et al. 2018). The dynamo drivers have not yet been systematically measured as a function of rotation for these simulations. In particular, we are interested in whether or not the $\alpha$ tensor becomes anisotropic in these simulations. These are the main purposes of the present paper.

We use the test-field method (Schrinner et al. 2005, 2007) to determine the turbulent transport coefficients. This method has been shown to give a good description of the dynamo processes in global dynamo simulations at moderate Reynolds numbers (Schrinner 2011; Schrinner et al. 2011, 2012; Warnecke et al. 2018; Warnecke 2018; Viviani et al. 2019). As the current testfield method only works for cases where the large-scale magnetic field is axisymmetric, we restrict our setup to azimuthal wedges of one-quarter of a sphere. Hence, large-scale nonaxisymmetric modes are suppressed and we can study the rotational dependency of the turbulent transport coefficients independently of other parameters. The dataset containing the turbulent transport coefficients as well as the mean flows for all runs is available online ${ }^{1}$.

\section{Model and setup}

We model the stellar convection zone in a spherical wedge $(r, \theta, \phi)$, with the same depth as the Sun, $(r=0.7 R$ to $r=R)$, where $R$ is the stellar radius. We restrict our domain to a quarter of a sphere $(0 \leq \phi \leq \pi / 2)$ without poles $\left(\theta_{0} \leq \theta \leq \pi-\theta_{0}\right.$, where $\left.\theta_{0}=15^{\circ}\right)$ for numerical reasons. We solve equations of magnetohydrodynamics in a fully compressible regime, including the induction equation for the magnetic field $\boldsymbol{B}$ in terms of the vector potential $\boldsymbol{A}$, which ensures the solenoidality of $\boldsymbol{B}=\boldsymbol{\nabla} \times \boldsymbol{A}$, the momentum equation in terms of the velocity $\boldsymbol{u}$, the continuity equation for the density $\rho$, and the energy equation in terms of the specific entropy $s$ together with an equation of state for an ideal gas with temperature $T$. Rotation is included via the Coriolis force $\boldsymbol{\Omega}_{0} \times \boldsymbol{u}$, where $\boldsymbol{\Omega}_{0}=\Omega_{0}(\cos \theta,-\sin \theta, 0)$ is the rotation vector with the bulk rotation $\Omega_{0}$, and gravity via Keplerian acceleration. The plasma is heated by a constant heat flux at the bottom of the convection zone and is cooled at the top by invoking a black-body boundary condition. We use a periodic boundary condition in the azimuthal direction for all quantities, a stress-free condition for the velocity on all other boundaries, and a perfect conductor condition for the magnetic field at the bottom radial and the latitudinal boundaries. At the top radial boundary, the magnetic field is radial. Further details of the model setup are described in Käpylä et al. (2013) and will not be repeated here.

Our model is characterized by non-dimensional input parameters: the normalized rotation rate and the resulting Taylor number,

$\tilde{\Omega}=\Omega_{0} / \Omega_{\odot}, \quad \mathrm{Ta}=\left[2 \Omega_{0}(0.3 R)^{2} / v\right]^{2}$,

where $\Omega_{\odot}=2.7 \times 10^{-6} \mathrm{~s}^{-1}$ is the rotation rate of the Sun, and $v$ is the constant kinematic viscosity, and the sub-grid-scale thermal and magnetic Prandtl numbers are

$\operatorname{Pr}_{\mathrm{SGS}}=\frac{v}{\chi_{\mathrm{m}}^{\mathrm{SGS}}}, \quad \operatorname{Pr}_{\mathrm{M}}=\frac{v}{\eta}$

where $\chi_{\mathrm{m}}^{\mathrm{SGS}}$ is the sub-grid-scale thermal diffusivity in the middle of the convection zone, and $\eta$ is the constant magnetic diffusivity. Additionally, we define a turbulent Rayleigh number calculated from a hydrostatic one-dimensional model

$\mathrm{Ra}=\frac{G M(0.3 R)^{4}}{v \chi_{\mathrm{m}}^{\mathrm{SGS}} R^{2}}\left(-\frac{1}{c_{\mathrm{P}}} \frac{\mathrm{d} s_{\mathrm{hs}}}{\mathrm{d} r}\right)_{(r=0.85 R)}$,

where $s_{\mathrm{hs}}$ is the specific entropy in the hydrostatic model, $G$ is the gravitational constant, $M$ is the mass of the star, and $c_{\mathrm{P}}$ is the specific heat capacity at constant pressure.

http://doi.org/10.5281/zenodo. 3629665 
Table 1. Summary of runs.

\begin{tabular}{|c|c|c|c|c|c|c|c|c|c|c|c|c|c|c|c|}
\hline Run & $\tilde{\Omega}$ & $\mathrm{Ta}\left[10^{6}\right]$ & $\mathrm{Ra}\left[10^{7}\right]$ & $\operatorname{Pr}_{S G S}$ & $\operatorname{Re}$ & Co & DR & $E_{\mathrm{kin}}^{\mathrm{tot}}$ & $E_{\text {kin }}^{\mathrm{dif}}$ & $E_{\mathrm{kin}}^{\mathrm{mer}}$ & $E_{\mathrm{kin}}^{\mathrm{flu}}$ & $E_{\mathrm{mag}}^{\mathrm{tot}}$ & $E_{\mathrm{mag}}^{\text {tor }}$ & $E_{\mathrm{mag}}^{\mathrm{pol}}$ & $E_{\mathrm{mag}}^{\mathrm{flu}}$ \\
\hline H0 & 0.0 & 0.0 & 4.0 & 2.0 & 52 & 0.0 & $?$ & 4.863 & 0.127 & 0.706 & 4.031 & & & & \\
\hline H0.005 & 0.005 & $1.3(-4)$ & 4.0 & 2.0 & 52 & 0.006 & $?$ & 4.884 & 0.124 & 0.677 & 4.083 & & & & \\
\hline H0.01 & 0.01 & $5.4(-4)$ & 4.0 & 2.0 & 52 & 0.011 & AS & 4.873 & 0.134 & 0.652 & 4.087 & & & & \\
\hline H0.1 & 0.1 & $5.4(-2)$ & 4.0 & 2.0 & 47 & 0.12 & AS & 10.307 & 6.727 & 0.293 & 3.298 & & & & \\
\hline H0.5 & 0.5 & 1.3 & 4.0 & 2.0 & 47 & 0.62 & AS & 11.203 & 7.608 & 0.292 & 3.303 & & & & \\
\hline H1 & 1.0 & 5.4 & 4.0 & 2.0 & 49 & 1.2 & AS & 54.087 & 50.976 & 0.554 & 2.557 & & & & \\
\hline H1.5 & 1.5 & 9.7 & 4.0 & 2.0 & 41 & 1.9 & AS & 62.036 & 60.103 & 0.337 & 2.364 & & & & \\
\hline $\mathrm{H} 2$ & 2.0 & 21.6 & 4.0 & 2.0 & 44 & 2.7 & AS & 14.515 & 11.699 & 0.051 & 2.765 & & & & \\
\hline $\mathrm{H} 2.5$ & 2.5 & 33.7 & 4.0 & 2.0 & 43 & 3.4 & $S$ & 9.541 & 6.811 & 0.041 & 2.689 & & & & \\
\hline H3.0 & 3.0 & 48.6 & 4.0 & 2.0 & 41 & 4.3 & S & 10.669 & 8.109 & 0.035 & 2.526 & & & & \\
\hline H5 & 5.0 & 125 & 4.0 & 2.0 & 34 & 8.4 & $S$ & 5.402 & 3.569 & 0.018 & 1.815 & & & & \\
\hline H7 & 7.0 & 190 & 3.4 & 2.4 & 26 & 13.6 & $\mathrm{~S}$ & 4.381 & 3.078 & 0.011 & 1.129 & & & & \\
\hline $\mathrm{H} 10$ & 10.0 & 260 & 2.8 & 2.9 & 18 & 22.9 & S & 3.107 & 2.176 & 0.005 & 0.926 & & & & \\
\hline M0.5 & 0.5 & 1.3 & 4.0 & 2.0 & 44 & 0.7 & AS & 7.141 & 3.910 & 0.199 & 3.032 & 0.362 & 0.032 & 0.019 & 0.311 \\
\hline M1 & 1.0 & 5.4 & 4.0 & 2.0 & 40 & 1.5 & AS & 4.084 & 1.259 & 0.074 & 2.751 & 0.775 & 0.074 & 0.063 & 0.638 \\
\hline M1.5 & 1.5 & 12 & 4.0 & 2.0 & 39 & 2.2 & AS & 3.163 & 0.691 & 0.045 & 2.427 & 0.789 & 0.107 & 0.077 & 0.605 \\
\hline M2 & 2.0 & 22 & 4.0 & 2.0 & 40 & 2.9 & AS & 3.065 & 0.483 & 0.036 & 2.547 & 0.479 & 0.055 & 0.048 & 0.376 \\
\hline M2.5 & 2.5 & 34 & 4.0 & 2.0 & 40 & 3.7 & $S$ & 2.992 & 0.524 & 0.029 & 2.438 & 0.506 & 0.087 & 0.044 & 0.375 \\
\hline M3 & 3.0 & 49 & 4.0 & 2.0 & 39 & 4.5 & $\mathrm{~S}$ & 3.584 & 1.268 & 0.026 & 2.290 & 0.593 & 0.120 & 0.050 & 0.423 \\
\hline M4 & 4.0 & 86 & 4.0 & 2.0 & 36 & 6.6 & $\mathrm{~S}$ & 3.741 & 1.741 & 0.019 & 1.981 & 0.801 & 0.144 & 0.100 & 0.557 \\
\hline M5 & 5.0 & 35 & 4.0 & 2.0 & 34 & 8.6 & S & 3.600 & 1.804 & 0.015 & 1.780 & 0.987 & 0.190 & 0.136 & 0.660 \\
\hline M7 & 7.0 & 264 & 4.0 & 2.0 & 31 & 13.4 & S & 2.481 & 1.040 & 0.009 & 1.432 & 1.109 & 0.206 & 0.198 & 0.704 \\
\hline M10 & 10.0 & 540 & 4.0 & 2.0 & 27 & 21.5 & $S$ & 1.550 & 0.465 & 0.005 & 1.079 & 1.159 & 0.212 & 0.242 & 0.705 \\
\hline M15 & 15.0 & 1897 & 7.4 & 2.0 & 27 & 40.3 & $S$ & 0.746 & 0.066 & 0.002 & 0.677 & 1.216 & 0.126 & 0.290 & 0.799 \\
\hline M30 & 30.0 & 13488 & 16.1 & 2.0 & 26 & 110.9 & S & 0.392 & 0.021 & 0.001 & 0.370 & 2.007 & 0.305 & 0.462 & 1.241 \\
\hline
\end{tabular}

Notes. Second to fourth columns: input parameters. Columns 6 to 15 show the output parameters, which are calculated from the saturated stage of the simulations. DR indicates the type of differential rotation, either it is anti-solar (AR), or solar (S)-like or inconclusive (?) differential rotation. The energies $E$ are given in $10^{5} \mathrm{~J} \mathrm{~m}^{-2}$ and their definitions are given in Eqs. (5)-(9). All runs have a density contrast of $\Gamma_{\rho} \equiv \rho(r=0.7 R) / \rho(R)=31$ and the MHD runs (Set M) $\operatorname{Pr}_{M}=1$. The resolution is $180 \times 256 \times 128$ grid points for all runs, except it is $360 \times 512 \times 256$ for Run H10.

We use the fluid and magnetic Reynolds numbers together with the Coriolis number

$\operatorname{Re}=\frac{u_{\mathrm{rms}}}{v k_{\mathrm{f}}}, \quad \operatorname{Re}_{\mathrm{M}}=\frac{u_{\mathrm{rms}}}{\eta k_{\mathrm{f}}}, \quad \mathrm{Co}=\frac{2 \Omega_{0}}{u_{\mathrm{rms}} k_{\mathrm{f}}}$

to characterize our simulations. Here, $k_{\mathrm{f}}=2 \pi / 0.3 R \approx 21 / R$ is an estimate of the wavenumber of the largest eddies in the convection zone and $u_{\mathrm{rms}}=\sqrt{(3 / 2)\left\langle u_{r}^{2}+u_{\theta}^{2}\right\rangle_{r \theta \phi t}}$ is the rms velocity and the subscripts indicate averaging over $r, \theta, \phi$ and a time interval covering the saturated state. These non-dimensional input and characteristic parameters are given in Table 1.

For our analysis we divide each field into a mean (axisymmetric) part and a fluctuating part, the mean being denoted with an overbar and the fluctuations with a prime, for example, $\boldsymbol{B}=\overline{\boldsymbol{B}}+\boldsymbol{B}^{\prime}$ and $\boldsymbol{u}=\overline{\boldsymbol{u}}+\boldsymbol{u}^{\prime}$. We note that this axisymmetric mean follows the Reynolds rules. As we are using the wedge approximation in the azimuthal direction, the large-scale nonaxisymmetric modes with azimuthal degrees of 1,2 , and 3 are suppressed. Hence, the adopted azimuthal mean can be reliably used to compute the mean fields, which accurately describes the large-scale magnetic field evolution. With this azimuthal mean, we define a $r$ - and $\theta$-dependent turbulent velocity as $u_{\mathrm{rms}}^{\prime}(r, \theta)=$ $\left\langle\overline{\boldsymbol{u}^{\prime 2}}\right\rangle_{t}^{1 / 2}$ and the corresponding turnover time of the convection $\tau_{\text {tur }}=H_{p} \alpha_{\mathrm{MLT}} / u_{\mathrm{rms}}^{\prime}$, where $H_{p}=-(\partial \ln \bar{p} / \partial r)^{-1}$ is the pressure scale height and $\alpha_{\mathrm{MLT}}=5 / 3$ is the mixing length parameter.

We define the total kinetic energy as

$E_{\mathrm{kin}}^{\mathrm{tot}}=\frac{1}{2}\left\langle\rho \boldsymbol{u}^{2}\right\rangle_{V}$, which can be decomposed into energies of the fluctuating velocities, the differential rotation, and the meridional circulation:

$E_{\mathrm{kin}}^{\mathrm{flu}}=\frac{1}{2}\left\langle\rho \boldsymbol{u}^{\prime 2}\right\rangle_{V}, \quad E_{\mathrm{kin}}^{\mathrm{dif}}=\frac{1}{2}\left\langle\rho{\overline{u_{\phi}}}^{2}\right\rangle_{V}$

and $E_{\mathrm{kin}}^{\mathrm{mer}}=\frac{1}{2}\left\langle\rho\left({\overline{u_{r}}}^{2}+{\overline{u_{\theta}}}^{2}\right)\right\rangle_{V}$,

where \langle\rangle$_{V}$ indicate a volume average. In a similar way, the total magnetic energy,

$E_{\mathrm{mag}}^{\mathrm{tot}}=\left\langle\frac{\boldsymbol{B}^{2}}{2 \mu_{0}}\right\rangle_{V}$

can be decomposed into energies of the fluctuating fields, and the toroidal and poloidal magnetic fields:

$E_{\mathrm{mag}}^{\mathrm{flu}}=\left\langle\frac{\boldsymbol{B}^{\prime 2}}{2 \mu_{0}}\right\rangle_{V}, \quad E_{\mathrm{mag}}^{\mathrm{tor}}=\left\langle\frac{\bar{B}_{\phi}^{2}}{2 \mu_{0}}\right\rangle_{V}$ and $E_{\mathrm{mag}}^{\mathrm{pol}}=\left\langle\frac{\bar{B}_{r}^{2}+\bar{B}_{\theta}^{2}}{2 \mu_{0}}\right\rangle_{V}$.

To determine the turbulent transport coefficients from our simulations, we use the test-field method (Schrinner et al. 2005, 2007; Brandenburg et al. 2008; Warnecke et al. 2018) with the new convention introduced in Viviani et al. (2019). In the testfield method, nine independent test fields are used to calculate how the flow acts on these fields to generate a small-scale magnetic field and therefore an electromotive force $\mathcal{E}$. It is important to note that these test fields do not have a feedback effect on the simulated hydromagnetic quantities, and are therefore only diagnostics of the system. With the electromotive forces for each test field at hand, we have a large enough set of equations to solve 
for the turbulent transport coefficients from the ansatz expanding the $\mathcal{E}$ in terms of the mean magnetic field (Krause \& Rädler 1980):

$\mathcal{E}=\boldsymbol{\alpha} \cdot \overline{\boldsymbol{B}}+\boldsymbol{\gamma} \times \overline{\boldsymbol{B}}-\boldsymbol{\beta} \cdot(\boldsymbol{\nabla} \times \overline{\boldsymbol{B}})-\boldsymbol{\delta} \times(\boldsymbol{\nabla} \times \overline{\boldsymbol{B}})-\boldsymbol{\kappa} \cdot(\boldsymbol{\nabla} \overline{\boldsymbol{B}})^{(s)}$,

where $(\nabla \overline{\boldsymbol{B}})^{(s)}$ is the symmetric part of the magnetic diffusivity tensor. Here we have neglected contributions of higher-than firstorder derivatives. Also, $\alpha$ and $\beta$ are two-rank tensors, $\gamma$ and $\delta$ are vectors, and $\boldsymbol{\kappa}$ is a three-rank tensor. These five coefficients can be associated with different turbulent effects important for the magnetic field evolution: the $\alpha$ effect (Steenbeck et al. 1966) can lead to field amplification via helical flows, for example in stratified convection influenced by rotation; the $\gamma$ effect describes turbulent pumping of the mean magnetic field in the same way as a mean flow; $\beta$ describes turbulent diffusion; and the $\delta$ effect, also known as the Rädler effect (Rädler 1969), can lead to dynamo action in the presence of other effects, for example the $\alpha$ effect or shear, although it alone cannot lead to the growth of magnetic energy (Brandenburg \& Subramanian 2005), and the $\kappa$ effect, the physical interpretation of which is currently unclear. However, the $\kappa$ effect can contribute, in theory, to both the amplification and diffusion of magnetic fields.

In most cases, we express the measured quantities in a nondimensional form by normalizing them appropriately. For example, we define $\alpha_{0}=u_{\mathrm{rms}}^{\prime} / 3$ and $\eta_{\mathrm{t} 0}=\tau_{\text {tur }} u_{\mathrm{rms}}^{\prime 2} / 3$ as normalizations for $\boldsymbol{\alpha}$ tensor, and $\boldsymbol{\beta}$ and $\boldsymbol{\delta}$ tensors, respectively. However, sometimes we transfer them into physical units by defining the unit system based on the solar rotation rate $\Omega_{\odot}=2.7 \times 10^{-6} \mathrm{~s}^{-1}$, solar radius $R=7 \times 10^{8} \mathrm{~m}$, density at the bottom of the convection zone $\rho(0.7 R)=200 \mathrm{~kg} / \mathrm{m}^{3}$, and $\mu_{0}=4 \pi \cdot 10^{-7} \mathrm{H} \mathrm{m}^{-1}$. All simulations including the test-field method were performed using the Pencil Code' ${ }^{2}$.

\section{Results}

All simulations were run to the saturated stage and then continued with the test-field method switched on for another 50-100 years. All the computed diagnostic quantities and plots shown below are obtained from this time interval. The hydrodynamic (HD) runs are labeled with an "H", and magnetohydrodynamic (MHD) ones with an "M", while the number in the run label represents these rotation rates normalized to the solar one, $\tilde{\Omega}$.

We aimed at keeping all input parameters the same and only changed the rotation rate as shown in Table 1, but for some runs this strategy partially failed. For Runs H7 and H10, we had to increase the viscosity to stabilize the simulations against strong shearing motions. For Runs M10 to M30 we decreased all diffusivities $\left(v, \eta, \chi_{\mathrm{m}}^{\mathrm{SGS}}\right)$ but kept the Prandtl numbers $\left(\operatorname{Pr}_{\mathrm{SGS}}, \operatorname{Pr}_{\mathrm{M}}\right)$ unchanged in order to have roughly constant Reynolds numbers (Re, $\mathrm{Re}_{\mathrm{M}}$ ).

The Rayleigh number for Run M5 is around 100 times the critical value (Warnecke et al. 2018). However, the critical Ra is known to increase as a function of rotation (Chandrasekhar 1961). Hence, our slower(faster) rotating runs can be expected to exceed the critical value of the onset of convection by a larger(smaller) margin than M5. This might not be the ideal modeling strategy; a better approach would be to fix the level of supercriticality in each run, but this is currently computationally too expensive for such a large parameter study. Furthermore, as verified by Warnecke et al. (2018) for a run similar to Run M5, we do not expect a small-scale dynamo to be operating in our simulations.

\footnotetext{
2 https://github.com/pencil-code/
}

Finally, referring back to our earlier studies, we note the following. Runs M0.5 to M10 have already been discussed in Warnecke (2018) to determine the dynamo cycle properties, but not all the turbulent transport coefficients were presented in that study. Run M5 is similar to Run I in Warnecke et al. (2014), Run A1 in Warnecke et al. (2016), Run D3 in Käpylä et al. (2017) and Warnecke et al. (2018), and Run $\mathrm{G}^{\mathrm{W}}$ in Viviani et al. (2018). Furthermore, Run M3 is similar to Run B1 in Warnecke et al. (2016) and Runs M10 and M15 are similar to Runs $\mathrm{I}^{\mathrm{W}}$ and $\mathrm{J}^{\mathrm{W}}$ of Viviani et al. (2018).

\subsection{Rotational influence on kinetic and magnetic energies}

First, we discuss how the different energies are influenced by rotation. As shown in Figs. 1a and b, the total kinetic energy increases for slow rotation, with a maximum for the runs with strong anti-solar differential rotation. For rapidly rotating cases, the kinetic energy drops strongly because of the rotational quenching of convection. For the MHD runs, we do not find a maximum during the anti-solar differential rotation phase, but rather exhibit a dip during the transition; otherwise they also fall off, as in the HD runs. For the HD runs, the kinetic energy is dominated by the differential rotation, the fluctuating fields contribute around 10-30\% for most of the runs, and the contribution of the meridional circulation is weak for all runs, in particular for high rotation. For MHD runs, the contribution of the fluctuating field is dominating, and energy related to differential rotation and meridional circulation becomes even weaker with higher rotation. This is consistent with the findings of Viviani et al. (2018), where the energy of the fluctuating field is also dominating the kinetic energy for most of the runs. This is why we see that relaxing the wedge assumption does not strongly influence the energy balance in the flow field itself; the most dominant factor is the inclusion or exclusion of the magnetic fields. In the MHD cases, the energy of the meridional flow and differential rotation decreases roughly linearly overall, whereas the energy of the fluctuating and total fields decreases with a less steep slope.

All magnetic energy contributions show a weak increase with rotation, as shown in Fig. 1c. The fluctuating field is also dominating the total energy here, whereas the contribution from mean fields $\left(E_{\mathrm{mag}}^{\mathrm{tor}}+E_{\mathrm{mag}}^{\mathrm{pol}}\right)$ increases from $10 \%$ for slow rotation to around $40 \%$ of the total magnetic energy for the highest rotation. This is mostly because the poloidal contribution becomes stronger for larger rotation, whereas the toroidal contribution remains roughly constant. Also the magnetic energy shows a small enhancement for the runs with anti-solar differential rotation, however this happens in only one run. This seems to be in contradiction to the work by Brandenburg \& Giampapa (2018), where they re-plotted the data of Karak et al. (2015), finding a stronger increase with decreasing Co than in the present study. However, in the original work of Karak et al. (2015), the change in the magnetic field as a function of rotation appears insignificant with respect to the large error bars. It is also noteworthy that there is a difference between how the Co is changed in Karak et al. (2015) and how it changes in our study: these latter authors change the thermal Prandtl number and therefore the strength of convection, in which case their Taylor number did not change, in contrast to our strategy. Furthermore, the setup of these latter authors is different from ours in terms of the radial extent being somewhat smaller. These differences also lead to a transition from anti-solar to solar-like differential rotation at a quite different Co.

The ratio of magnetic energy to kinetic energy gives an indication for the dynamo efficiency for each run; see Fig. 1d. We 

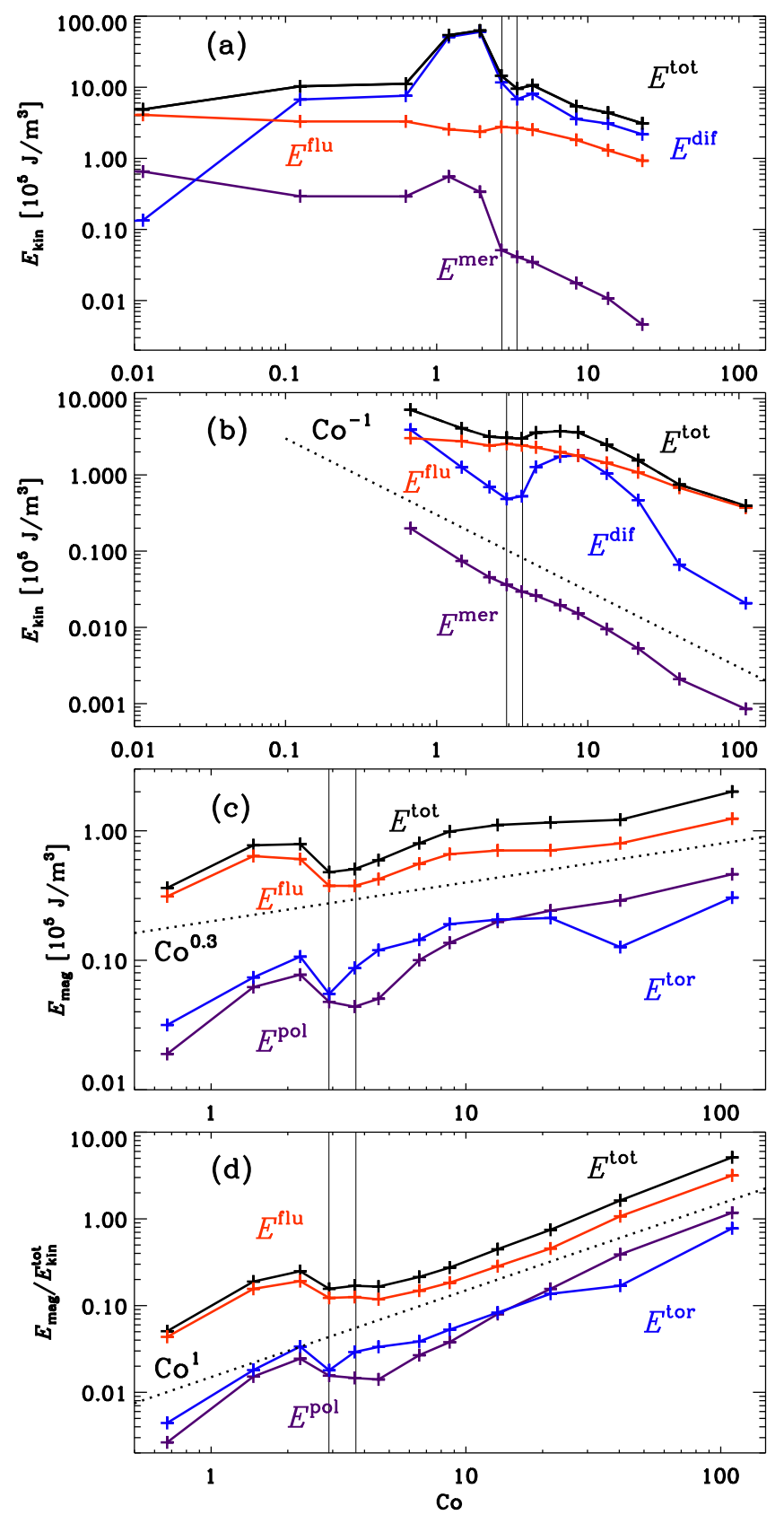

Fig. 1. Dependence of kinetic and magnetic energies on rotation in terms of Coriolis number Co. We show the total kinetic energy $E_{\text {kin }}^{\text {tot }}$ (black lines), which is composed of the energy of the fluctuating flows $E_{\mathrm{kin}}^{\mathrm{flu}}$ (red lines), the differential rotation $E_{\mathrm{kin}}^{\mathrm{dif}}$ (blue lines), and the meridional circulation $E_{\mathrm{kin}}^{\mathrm{mer}}$ (purple lines) for the HD runs (Set $\mathrm{H}$ ) in panel $a$ and for MHD runs (Set M) in panel $b$. Additionally, we show the total magnetic energy $E_{\text {mag }}^{\text {tot }}$ (black line) composed of the energy of the fluctuating magnetic field $E_{\mathrm{mag}}^{\text {flu }}$ (red), of the toroidal $E_{\mathrm{mag}}^{\text {tor }}$ (blue), and poloidal magnetic field $E_{\text {mag }}^{\mathrm{pol}}$ (purple) in panel $c$ and normalized by total kinetic energy $E_{\text {mag }}^{\text {tot }}$ in panel $d$. The dotted lines show the following relations between energies and Coriolis number: $E_{\mathrm{kin}} \propto \mathrm{Co}^{-1}$ in panel $b$, $E_{\mathrm{mag}} \propto \mathrm{Co}^{0.3}$ in panel $c$, and $E_{\mathrm{mag}} / E_{\mathrm{kin}} \propto \mathrm{Co}^{1}$ in panel $d$. The transition from anti-solar to solar-like differential rotations occurs in between the vertical lines (left line: last anti-solar run, right line: first solar-like run). See Eqs. (5)-(9) for the definition of the energies.

find that this ratio increases roughly linearly with rotational influence on covection, measured by Co. However, this is not only due to the fact that the kinetic energy decreases; the increase in magnetic energy as seen in Fig. 1c also plays a role. However, the decrease in kinetic energy seems to be the dominant behavior here. This is consistent with the findings of Viviani et al. (2018), where their Fig. 8 shows a linear tendency, but with a larger spread, and the work of Augustson et al. (2019), in which the authors collect data from a variety of simulations and also find a linear dependency with Co. We note here that the MHD runs are probably a more realistic representation of stars, as they have magnetic fields with similar strengths to those in our models. Hence, the dominance of the energy contribution of the differential rotation in HD, in particular in the anti-solar differential rotation cases, might be an artifact.

Comparing these modeling results with observations of stellar magnetic activity reveals fundamental differences. X-ray luminosity (e.g., Pizzolato et al. 2003; Wright \& Drake 2016), Ca II H\&K emission (e.g., Brandenburg et al. 1998), and surface magnetic field measurements using either Zeeman-Doppler imaging (ZDI; e.g., Vidotto et al. 2014) or Doppler imaging (e.g., Saar 2001) show an increase with Co with a power of around one or two for Co $\lesssim 10$. Our models show a weak increase of the magnetic energy with a power of around 0.3 over all runs; see Fig. 1c. This corresponds to an increase of around 0.15 in terms of the magnetic field strengths. This is in conflict with the observational results in two respects. First of all, the increase of magnetic field strength with Co is too low to be comparable with observations by a factor of between four and ten. Second, our models do not reproduce any two-dependency behavior, where for slow and moderate rotation $(\mathrm{Co} \lesssim 10)$ the magnetic field increases with rotation and for rapid rotation $(\mathrm{Co} \gtrsim 10)$ the energy is independent of rotation. Our results are only consistent with the latter behavior as our magnetic energy is only weakly increasing with Co. However, our models show this behavior already for slow and moderate rotation. One of the reasons for this discrepancy is the rotational dependence of supercriticality of convection (Chandrasekhar 1961). Because our Rayleigh number remains mostly constant, the convection is highly quenched by rotation as seen in the rotational dependence of the kinetic energy; see Fig. 1b. In real stars, the supercriticality is so high that the rotational quenching will not be important. Hence, the kinetic energy will be most likely independent of rotation. However, even if we take this into account and use the ratio of kinetic to magnetic energy as a function of Co to calculate the increase in magnetic field strength with Co, our models are still wrong by a factor of between two and four.

Furthermore, we want to note that even if the magnetic field strength increases only mildly as a function of rotation, its surface topology can influence coronal X-ray emission. Higher rotation leads to more helical fields, as it is also shown in Sect. 3.3, and this in turn can lead to higher X-ray luminosity as shown by Warnecke \& Peter (2019). Therefore, a weak increase in magnetic field strength might not be in contradiction with the strong increase in X-ray luminosity as a function of rotation for small and moderate rotation.

\subsection{Differential rotation and shear}

As already mentioned in the previous section, the differential rotation is strongly affected by rotation. As shown in Fig. 2, for no or very slow rotation (Runs H0 and H0.005) the differential rotation is very weak showing an inconclusive pattern; see also Table 1 and an overview of all differential rotation profiles in Figs. A.1 and A.2. For slow to moderate rotation $(\tilde{\Omega}=0.01$ to 2.0$)$, we find anti-solar differential rotation. For runs with higher rotation, this switches to solar-like differential rotation, where the equator rotates faster than the poles. 


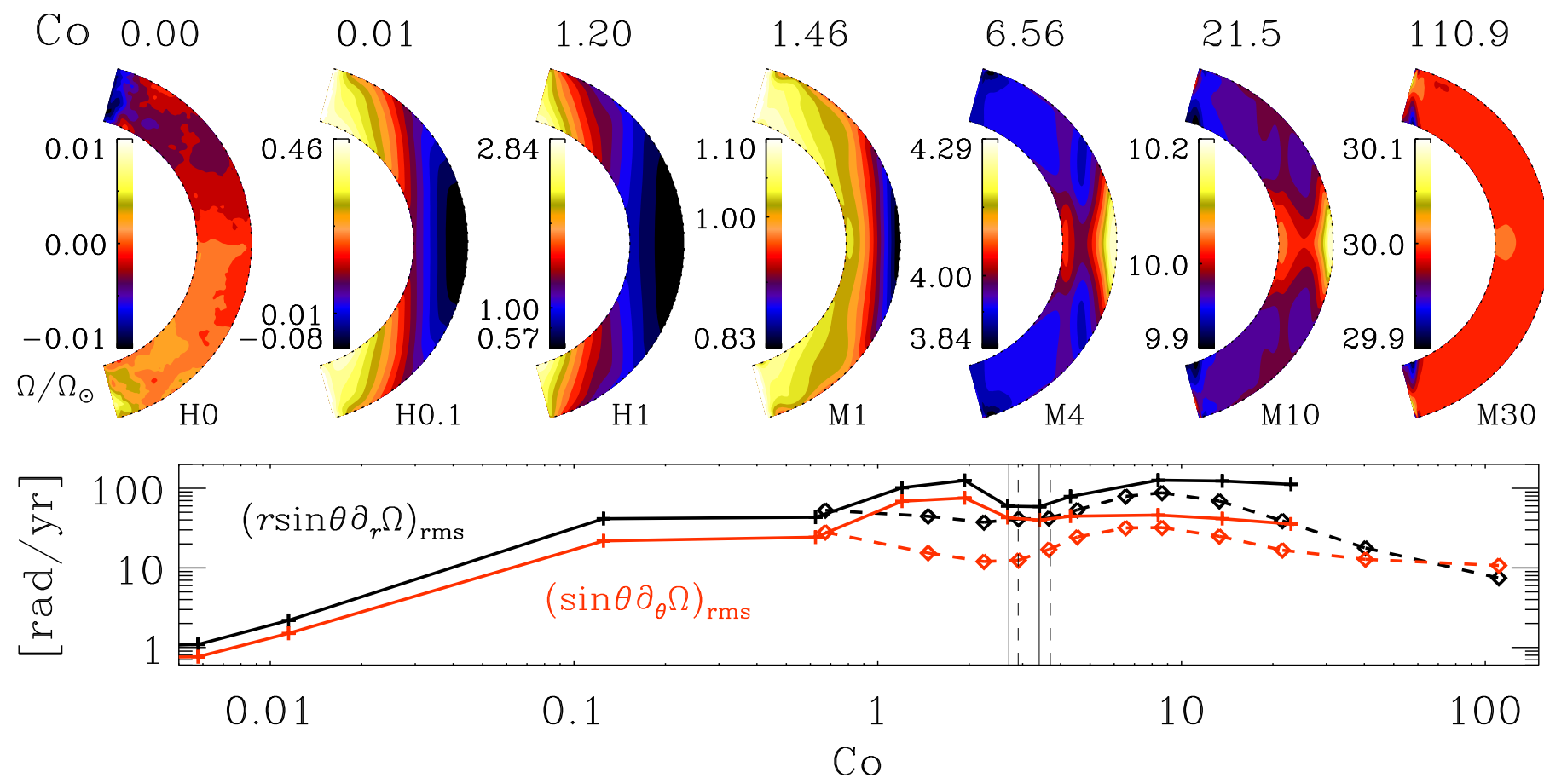

Fig. 2. Normalized local rotation profiles $\Omega / \Omega_{\odot}$ with $\Omega=\Omega_{0}+\overline{u_{\phi}} / r \sin \theta$ for Runs H0, H0.1, H1, M1, M4, M10, M30 and the rms values of the radial $r \sin \theta \partial \Omega / \partial r$ (black line) and latitudinal shear $\sin \theta \partial \Omega / \partial \theta$ (red) versus Coriolis number Co. The values have been calculated as a time average over the saturated state, and we have omitted the 5 closest grid points to the latitudinal boundary to remove boundary effects from the rms. The values of the HD runs (Set H) are shown with a solid line with plusses and the MHD runs (Set M) with dashed line with diamonds. The zero rotation run has been moved to $\mathrm{Co}=10^{-4}$ to be visible in the lower panel. The transition from anti-solar to solar-like differential rotations occurs in between the vertical lines (left line: last anti-solar run, right line: first solar-like run) and is shown as solid (Set H) and dashed (Set M) lines.

This transition has already been found in previous studies of stellar and planetary dynamos (Gastine et al. 2014; Käpylä et al. 2014; Fan \& Fang 2014; Featherstone \& Miesch 2015; Karak et al. 2015; Viviani et al. 2018). For rapidly rotating runs, the differential rotation becomes very weak and mostly pronounced at the equator and at the latitudinal boundaries.

As we are mostly interested in the analysis of the dynamo drivers in these runs, we focus next on how the shear acting on the magnetic field changes with rotation rate. As shown in the lower panel of Fig. 2, the rms value of the latitudinal and radial shear averaged over the whole convection zone increases for slow rotation when the differential rotation builds up. For the $\mathrm{HD}$ runs (Set $\mathrm{H}$ ), this value has a maximum for the antisolar differential rotation runs, and decreases during the differential rotation transition. The radial differential rotation also has a maximum in the solar-like differential rotation regime, while the latitudinal one remain roughly constant. For all HD runs, the radial differential rotation is 1.5 to 3 times larger than the latitudinal one, where the largest differences are seen for the rapidly rotating runs. For the MHD runs (Set M), the latitudinal shear is roughly independent from rotation rate, while the radial shear increases for the solar-like differential rotation cases and decreases for rapidly rotating runs. Also, for the MHD runs the radial shear is stronger than the latitudinal one, except for the runs with $\mathrm{Co}=20$ and larger. Hence, the weak increase of magnetic energy with rotation cannot be explained by an increase in shear, as the shear either remains constant or declines for large rotation rates.

\subsection{Rotational dependency of $\alpha \mathrm{s}$}

Now, we investigate the rotational dependence of $\boldsymbol{\alpha}$ and first focus on its general properties. We compute the trace of the $\alpha$ tensor, $\alpha_{\mathrm{tr}}=\alpha_{r r}+\alpha_{\theta \theta}+\alpha_{\phi \phi}$ using the test-field method. For comparison, we also calculate $\alpha$ based on the kinetic and current helicity, following Steenbeck et al. (1966) and Pouquet et al. (1976):

$\alpha_{\mathrm{K}}=-\frac{\tau_{\text {cor }}}{3} \overline{\boldsymbol{\omega}^{\prime} \cdot \boldsymbol{u}^{\prime}}, \quad \alpha_{\mathrm{M}}=\frac{\tau_{\text {cor }}}{3} \overline{\boldsymbol{j}^{\prime} \cdot \boldsymbol{b}^{\prime}} / \bar{\rho}, \quad \alpha_{\mathrm{KM}} \equiv \alpha_{\mathrm{K}}+\alpha_{\mathrm{M}}$,

where $\alpha_{\mathrm{K}}$ and $\alpha_{\mathrm{M}}$ are the kinetic and magnetic $\alpha$ coefficients, respectively, $\omega^{\prime}=\nabla \times \boldsymbol{u}^{\prime}$ is the fluctuating vorticity, $\overline{\omega^{\prime} \cdot \boldsymbol{u}^{\prime}}$ is the azimuthally averaged small-scale kinetic helicity, $\boldsymbol{j}^{\prime}=\boldsymbol{\nabla} \times \boldsymbol{b}^{\prime} / \mu_{0}$ is the fluctuating current density, $\overline{\boldsymbol{j}^{\prime} \cdot \boldsymbol{b}^{\prime}}$ is the azimuthally averaged small-scale current helicity, $\bar{\rho}$ is the mean density, and $\tau_{\text {cor }}$ is the turbulent correlation time, which we now set equal to the convective turn-over time, $\tau_{\text {cor }}=\tau_{\text {tur }}$. In Fig. 3, we show the meridional profiles and the rms values of the $\alpha_{\text {tr }}$ computed both from the HD (Set H) and MHD (Set M) runs, together with $\alpha_{\mathrm{K}}$ or $\alpha_{\mathrm{KM}}$, respectively, as a function of Co. We find that $\alpha_{\mathrm{tr}}$ from HD runs closely follows $\alpha_{\mathrm{K}}$ for slow and moderate rotation $(\mathrm{Co}=0$ to 4 ) in distribution and amplitude. $\alpha_{\text {tr }}$ from MHD runs is somewhat weaker than the other quantities. However, all the different measurements show very similar spatial distributions, and all show growth consistent with $\mathrm{Co}^{0.5}$. The magnetic part, $\alpha_{\mathrm{M}}$, is an order of magnitude weaker than $\alpha_{\mathrm{K}}$, but is growing with a similar power law as a function of rotation. $\alpha_{\mathrm{tr}}, \alpha_{\mathrm{K}}$ and $\alpha_{\mathrm{KM}}$ show profiles that are positive(negative) in the northern hemisphere in the upper(lower) part of the convection zone; the signs are opposite in the southern hemisphere. This spatial pattern seems to be roughly independent of rotation for $\alpha_{\mathrm{tr}} \mathrm{s}$ in this moderate rotation regime.

For higher rotation, $\alpha_{\mathrm{tr}}$ and $\alpha_{\mathrm{KM}}$ significantly decouple, and the HD and MHD test-field results start following each other tightly. While $\alpha_{\mathrm{KM}}$ and $\alpha_{\mathrm{K}}$ still continue growing with the same 


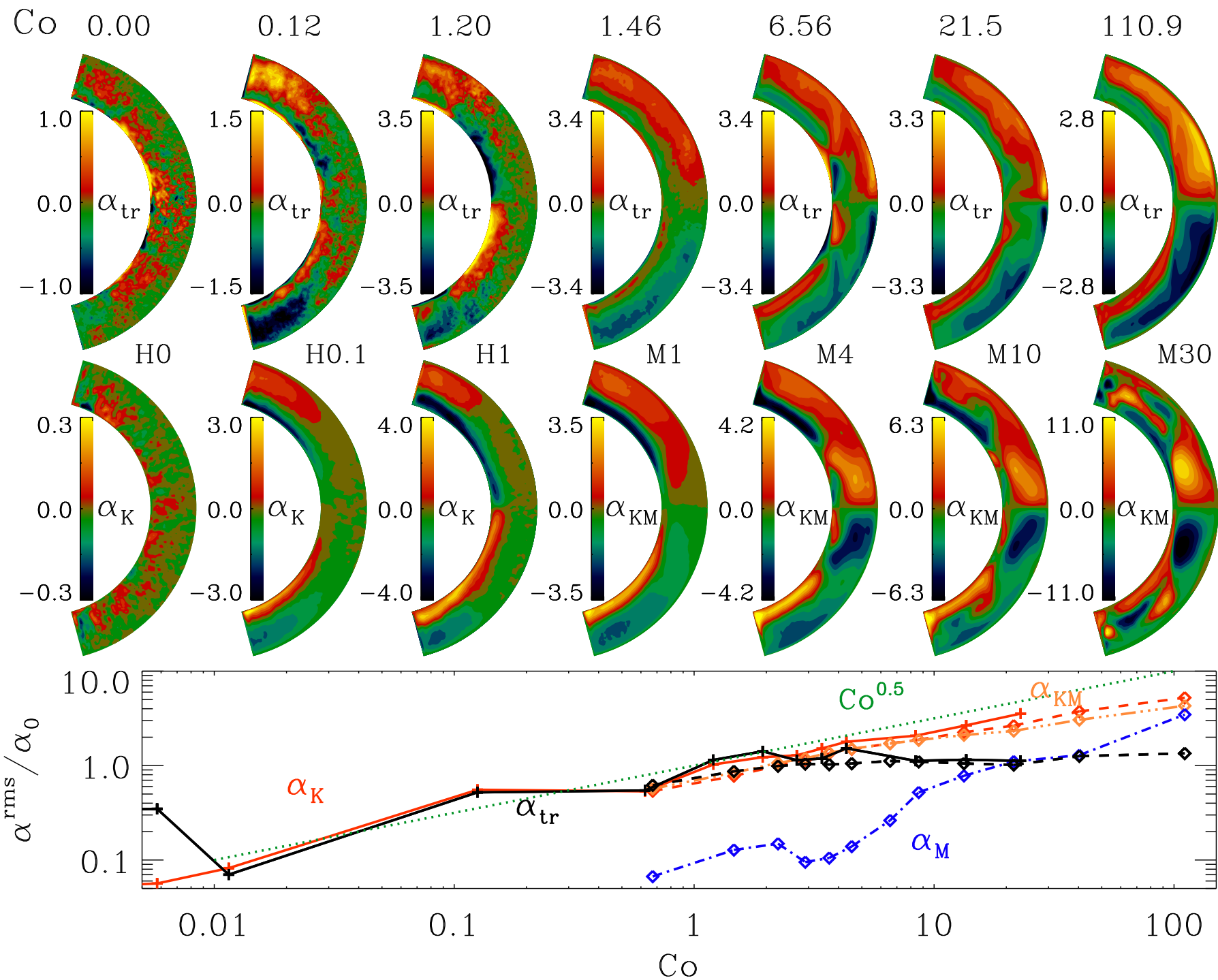

Fig. 3. Rotational dependence of the $\alpha$ profiles calculated with test-field method and calculated from helicities. In the first row, we plot the traces of the $\alpha$ tensors, $\alpha_{\mathrm{tr}}$, which were determined using the test-field method for a selection of HD (Set H) and MHD runs (Set M). In the second row, we plot the kinetic $\alpha_{\mathrm{K}}$ and the sum of kinetic and magnetic alpha $\alpha_{\mathrm{KM}}$ for the corresponding runs of Set $\mathrm{H}$ and Set M, respectively. In the last row, we show the root-mean-square values of the trace of the $\alpha$ tensor, $\alpha_{\text {tr }}$ (black lines), the kinetic $\alpha_{\mathrm{K}}$ (red), the magnetic $\alpha_{\mathrm{M}}$ (blue), and their sum $\alpha_{\mathrm{KM}}$ (orange) for all runs. The values of the HD runs are shown with a solid line and crosses, whereas the MHD runs are shown with a dashed line with diamonds. The greed dotted line indicates a power law with an exponent of 0.5 . All values are normalized by $\alpha_{0}=u_{\mathrm{rms}}^{\prime} / 3$. The zero rotation run has been moved to $\mathrm{Co}=10^{-4}$ to be visible in the lower panel.

power law as in the moderate rotation regime, both the test-fieldmeasured quantities no longer depend on rotation. Here, $\alpha_{\mathrm{M}}$ shows a much stronger dependence on rotation $(\propto \mathrm{Co})$ than any of the other quantities, and becomes comparable to $\alpha_{\mathrm{K}}$ for the most rapidly rotating case. This difference in between the theoretical prediction and test-field measurements could be explained by us not modeling the rotational dependence of $\tau_{\text {cor }}=\tau_{\text {tur }}$ correctly. The convective scale entering the calculation of $\tau_{\text {tur }}$ is known to be dependent on rotation. The theoretical calculation of Chandrasekhar (1961) predicts a dependence of $\mathrm{Co}^{-0.3}$, while the models of Featherstone \& Hindman (2016) and Viviani et al. (2018) show a dependence of $\mathrm{Co}^{-0.5}$. If we take this into account, the increase of $\alpha_{\mathrm{KM}}$ mostly vanishes.

Also the strongly growing $\alpha_{\mathrm{M}}$ contributes to the increase of $\alpha_{\mathrm{KM}}$. In our most rapidly rotating runs $\alpha_{\mathrm{M}}$ can, locally, even exceed the value of $\alpha_{\mathrm{K}}$, as is evident from Fig. 4. We see that $\alpha_{\mathrm{M}}$ is mostly negative (positive) in the northern (southern) hemi- sphere in the upper part of the convection zone, and positive (negative) below, and therefore it has the opposite sign compared to $\alpha_{\mathrm{K}}$. The peak values of $\alpha_{\mathrm{M}}$ are larger than $\alpha_{\mathrm{K}}$ for rapidly rotating runs, but these locations are not those where $\alpha_{\mathrm{K}}$ is the strongest. This leads to a more complicated distribution of $\alpha_{\mathrm{KM}}$, where at high latitudes in the middle of the convection zone, the sign of $\alpha_{\mathrm{KM}}$ changes due to $\alpha_{\mathrm{M}}$, but at low latitudes $\alpha_{\mathrm{KM}}$ is still dominated by $\alpha_{\mathrm{K}}$. Hence, in the most rapidly rotating cases, the $\alpha_{\mathrm{KM}}$ profiles no longer closely match with the test-field measured profiles. The formula of Eq. (11) has been introduced by Pouquet et al. (1976) for cases where $\alpha_{\mathrm{M}}$ is small and acts as a perturbation to $\alpha_{\mathrm{K}}$. In our case, in contrast, $\alpha_{\mathrm{M}}$ is even stronger than $\alpha_{\mathrm{K}}$ at some locations, so we cannot expect this expression to be valid in this regime. One possible inconsistency in our approach is to regard the correlation times, $\tau_{\text {cor }}$, of the kinetic and magnetic parts of the $\alpha$ effect as equal. In reality, this might not be the case, and our analysis should be refined. In any case, 

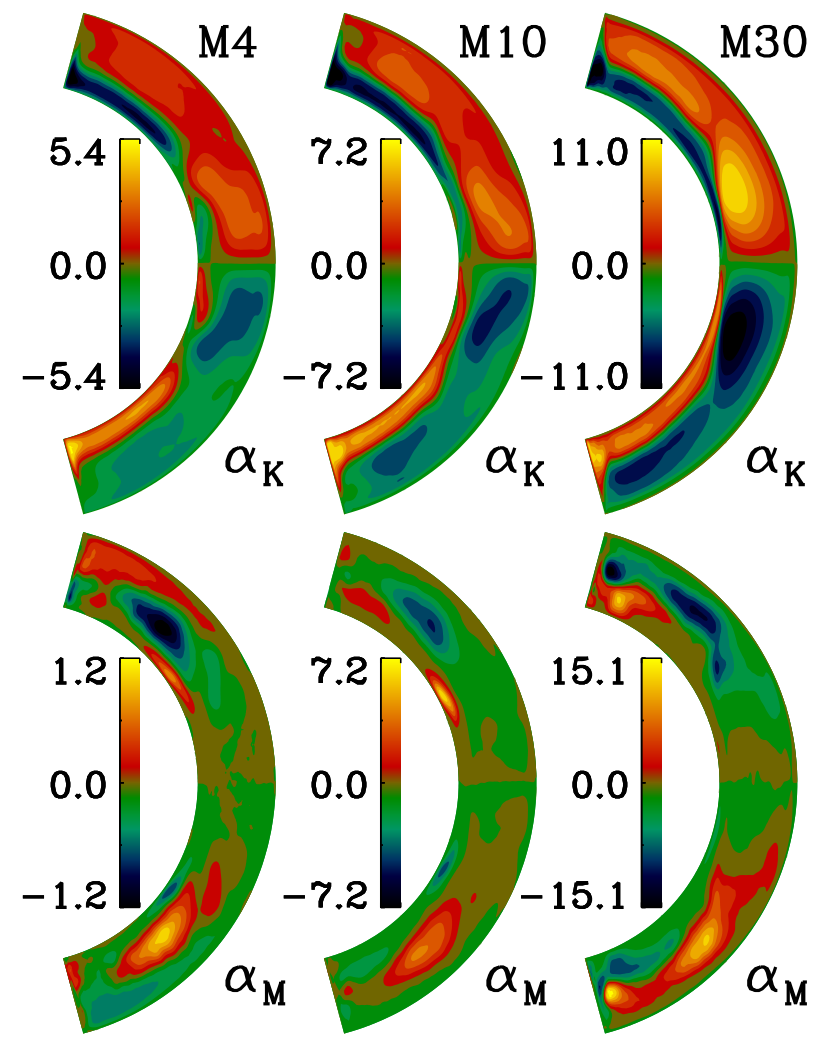

Fig. 4. Kinetic $\alpha_{\mathrm{K}}$ and magnetic $\alpha_{\mathrm{M}}$ for three of the runs shown in Fig. 3 , which have a significant contribution from $\alpha_{\mathrm{M}}$. All values are normalized by $\alpha_{0}=u_{\mathrm{rms}}^{\prime} / 3$.

our current results show that $\alpha_{\mathrm{KM}}$, using the procedure adopted here and very commonly by other authors analyzing their MHD simulations, should be only used as a proxy of the $\alpha$ effect with some caution.

In summary, we find from our simulations that quenching of the $\alpha$ effect in terms of rotation can be mostly explained by the changes in the turbulent correlation length. In addition, we note that, due to the increasing ratio of magnetic to kinetic energy as a function of rotation, we may also be seeing magnetic quenching reducing the $\alpha$ effect. By comparing the HD and MHD test-field measurements, however, we obtain very similar rms values in the regime where the magnetic field should be dominant. Hence, the magnetic quenching seems to be weak in these particular runs. However, even if the differences in the rms values can be small, locally there can be strong differences between the HD and the MHD runs, as found by Warnecke et al. (2018).

According to the Parker-Yoshimura rule (Parker 1955; Yoshimura 1975), an $\alpha \Omega$ dynamo will produce an equatorward migrating dynamo wave if $\alpha_{\phi \phi}$ and radial shear have different signs on opposite hemispheres. Typically, convection simulations produce positive $\alpha_{\phi \phi}$ in the north, while the radial shear is weak and positive in the bulk of the convection zone. There is often a narrow layer of reversed sign of $\alpha_{\phi \phi}$ in the bottom of the convection zone, as is also the case in the simulations presented here, but this is not large enough to contribute to the correct migratory properties of the wave. Instead, equatorward migration is driven by an additional local region of negative radial shear together with the positive $\alpha_{\phi \phi}$ in the bulk. Only in the thicker shell simulations in the planetary context has there been success in producing a thick-enough layer of reversed sign of helicity to drive the equatorward dynamo wave with a pos-
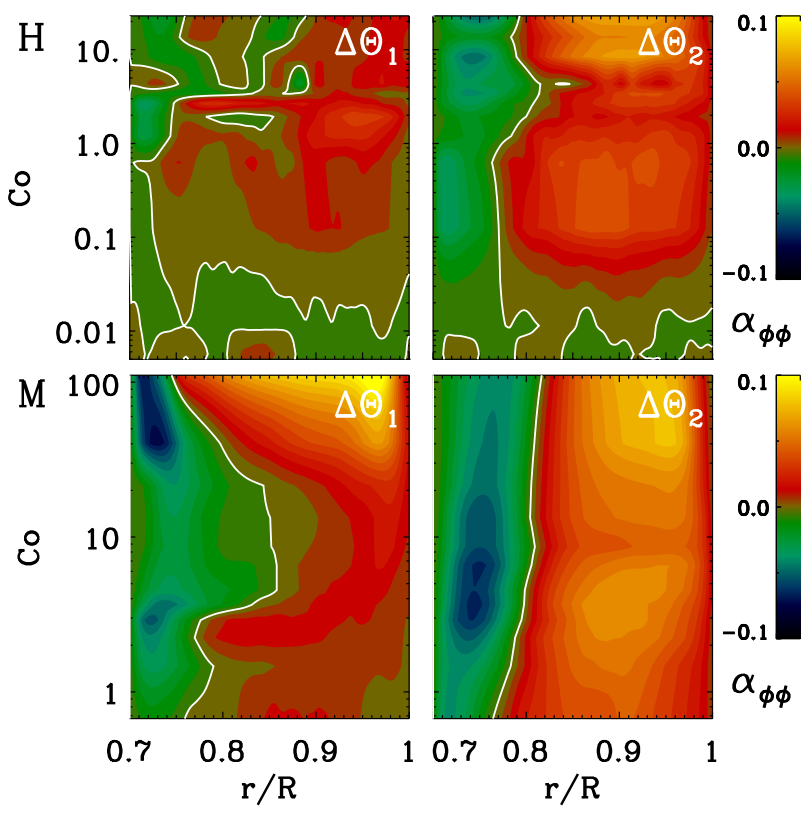

Fig. 5. Radial inversion of $\alpha_{\phi \phi}$ as a function of Co for two latitudinal strips for the HD runs (Set $\mathrm{H}$ ) (top row) and MHD runs (Set M) (bottom row). In the first column we average $\alpha_{\phi \phi}$ at low latitudes, i.e., $\Delta \Theta_{1}=10^{\circ}-20^{\circ}$, and in the second column we average over mid latitudes $\Delta \Theta_{2}=50^{\circ}-60^{\circ}$. The zero values are indicated with a white line. All values are normalized by $\alpha_{0}=u_{\mathrm{rms}}^{\prime} / 3$.

itive radial shear (Duarte et al. 2016). To investigate how the thickness of the inversion layer changes as a function of rotation, we plot in Fig. 5 the radial distribution of $\alpha_{\phi \phi}$ for two latitudinal bands at low $\left(\Delta \Theta_{1}=10^{\circ}-20^{\circ}\right)$ and mid latitudes $\left(\Delta \Theta_{2}=50^{\circ}-60^{\circ}\right)$. At low latitudes, the HD runs do not show an inversion layer at all, but at the higher latitude band, an inversion layer extending roughly one fourth of the convection zone is visible, becoming somewhat wider as a function of rotation. However, the magnitude of $\alpha_{\phi \phi}$ in this layer is very weak. In the MHD runs, $\alpha_{\phi \phi}$ is always negative in the lower fourth of the convection zone, but for values of Co of between 4 and 30 this region reaches up to half of the convection zone at low latitudes. Increasing rotation even more, the inversion layer again becomes narrower. For the mid latitudes, the region of negative $\alpha_{\phi \phi}$ is also located in the lower third of the convection zone. We find a tendency for this region to increase for larger rotation. Here, $\alpha_{\phi \phi}$ is stronger in the inversion layer in the MHD cases than in the HD ones. Hence, we do not find inversion layers extending close to the surface from our simulations, as is found by Duarte et al. (2016).

\subsection{Anisotropy of the $\alpha$ tensor}

As a next step, we further investigate the $\boldsymbol{\alpha}$ tensor by looking at each of the diagonal components. For this, we show in Fig. 6 their meridional profiles and the rms values. For slow rotation, until $\mathrm{Co}=0.7$, the diagonal components have similar strengths, but for larger rotation their behaviors diverge. Here, $\alpha_{r r}$ shows a distribution with positive(negative) values in the upper part and negative(positive) values in the bottom of the convection zone in the northern(southern) hemisphere; it has the strongest values at low latitudes and near the surface. Here, $\alpha_{r r}$ is the dominating component for moderate rotation $(\mathrm{Co}=1$ to 11$)$, in particular in the HD runs. For the highest rotation rates (Runs M10 to M30), 
Co 0.00
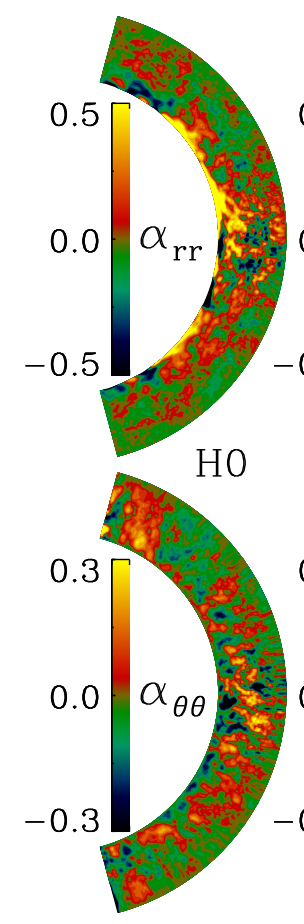

.

0.12

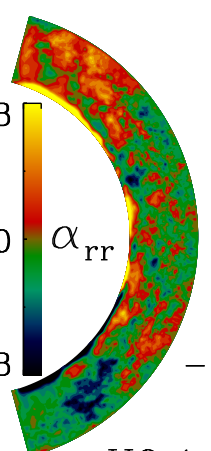

HO. 1

0.6

0.6
1.20

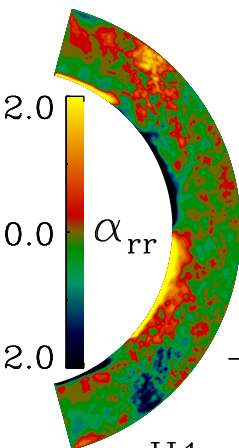

$\mathrm{H} 1$
1.46

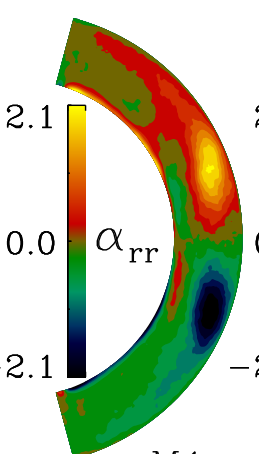

M1
6.56

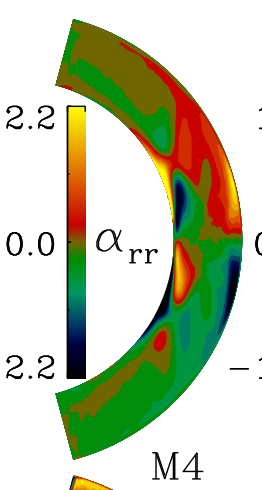

21.5

110.9

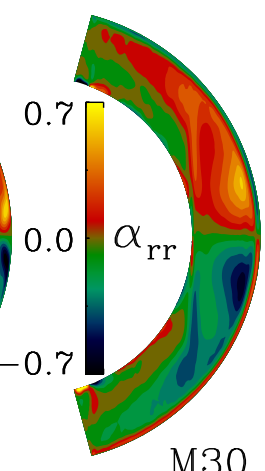

M10
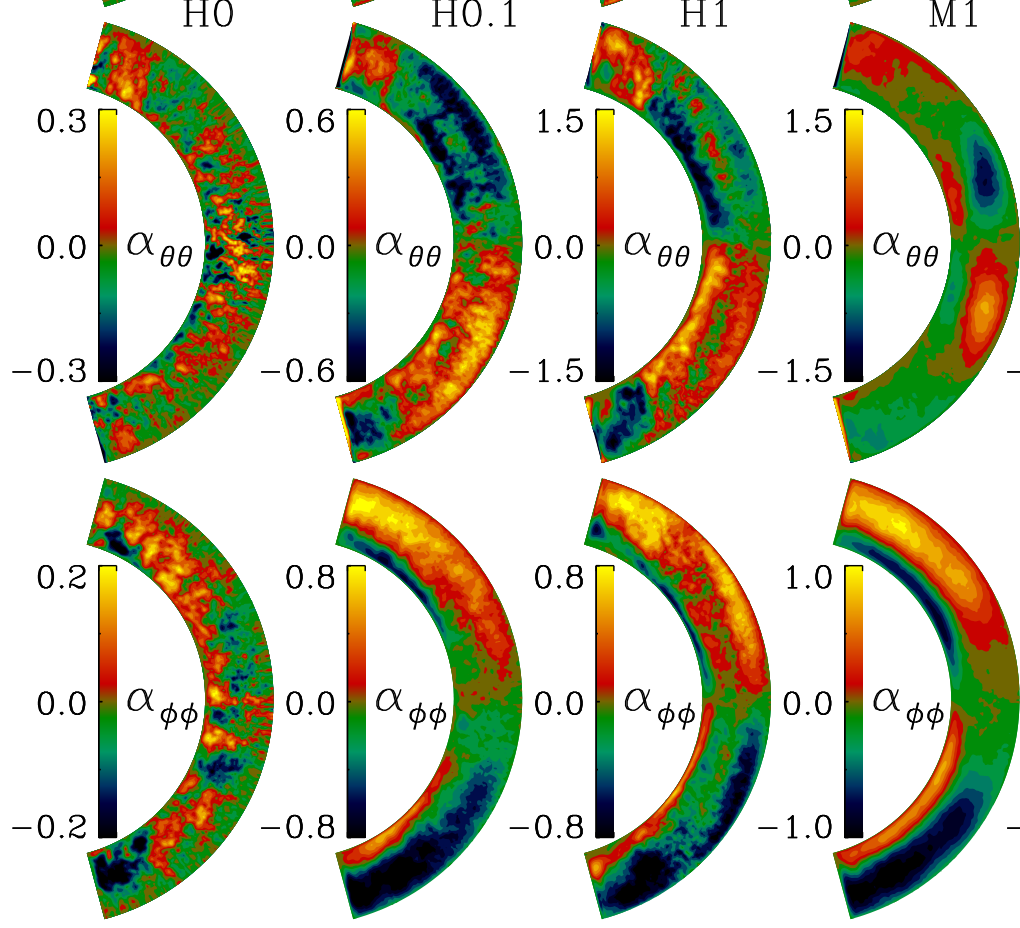

M30
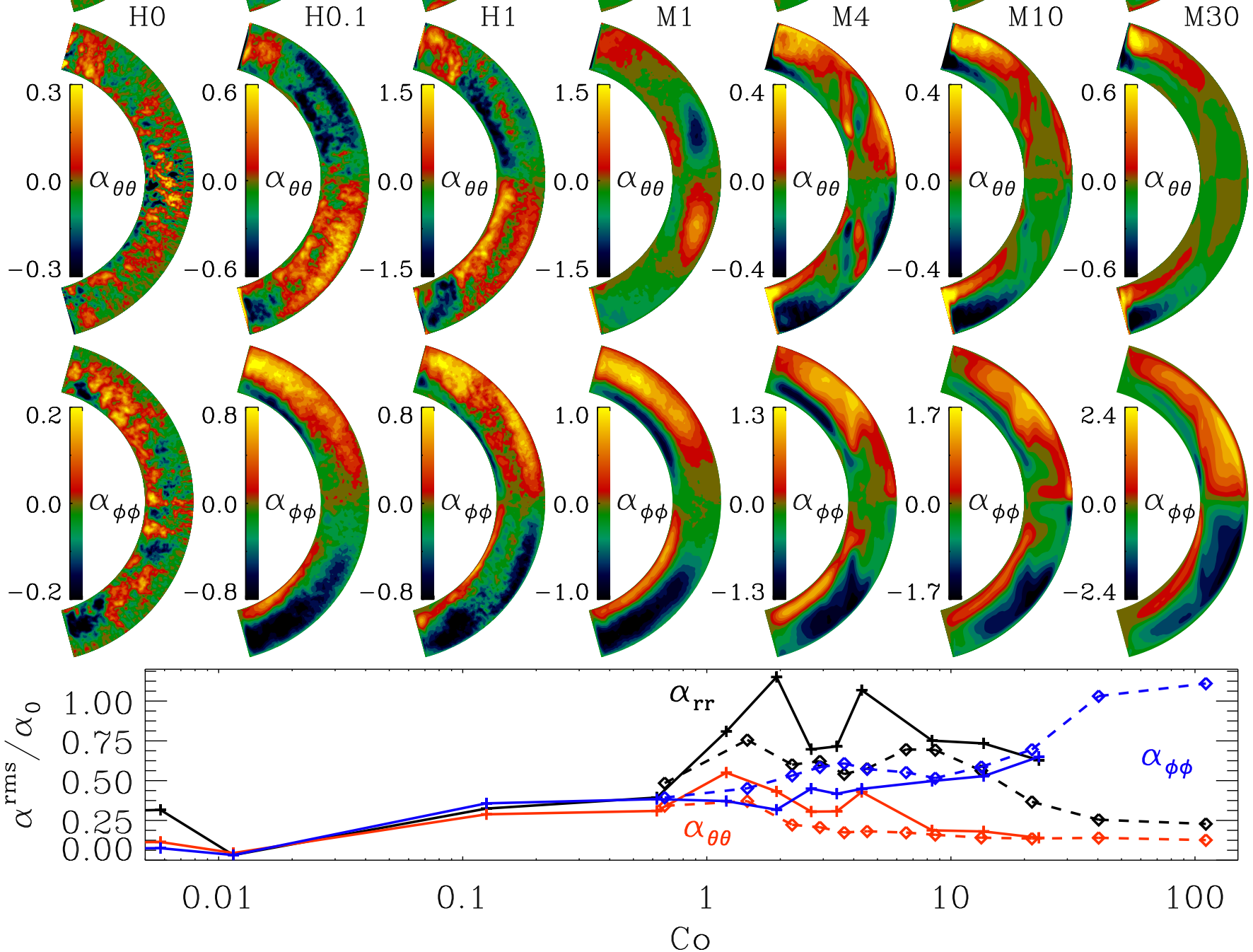

Fig. 6. Rotational dependency of the diagonal $\alpha$ components. We show meridional profiles of $\alpha_{r r}$ (top row), $\alpha_{\theta \theta}$ (second row), and $\alpha_{\phi \phi}$ (third row), and their rms values (bottom row), with $\alpha_{r r}$ (black lines), $\alpha_{\theta \theta}$ (red), and $\alpha_{\phi \phi}$ (blue). As in Fig. 3 the values of the HD runs (Set H) are shown with a solid line and crosses, whereas the MHD runs (Set M) are shown with a dashed line with diamonds. All values are normalized by $\alpha_{0}=u_{\mathrm{rms}}^{\prime} / 3$. The zero rotation run has been moved to $\mathrm{Co}=10^{-4}$ to be visible in the lower panel.

we find a thin layer of opposite sign at the surface. However, in this regime $\alpha_{r r}$ becomes very weak.

Similarly to $\alpha_{r r}, \alpha_{\theta \theta}$ has its strongest values for moderate rotation $(\mathrm{Co}=1$ to 11$)$ with larger values in the HD runs, but it remains subdominant to the other two diagonal components at all rotation rates. Interestingly, $\alpha_{\theta \theta}$ is the only diagonal component, which changes sign as a function of rotation: for Co $<1.5, \alpha_{\theta \theta}$ is dominantly negative(positive) in the northern(southern) hemisphere at low to mid latitudes. For $\mathrm{Co}>1.5$, it becomes positive (negative), and finally approaches zero, being weaker than $\alpha_{r r}$ for the highest rotation rates. This change was also reported by Viviani et al. (2019) by comparing their run with $\mathrm{Co}=2.8$ to the run of Warnecke et al. (2018) with $\mathrm{Co}=8.3$. At high latitudes, the distribution is similar as for $\alpha_{\mathrm{K}}$.

We find that $\alpha_{\phi \phi}$ follows the sign distribution of $\alpha_{\mathrm{K}}$ for all values of rotation, except for zero rotation. Its maximum values move from high to low latitudes as the rotations becomes stronger, similarly to $\alpha_{r r}$. An interesting finding is that, while $\alpha_{r r}$ 


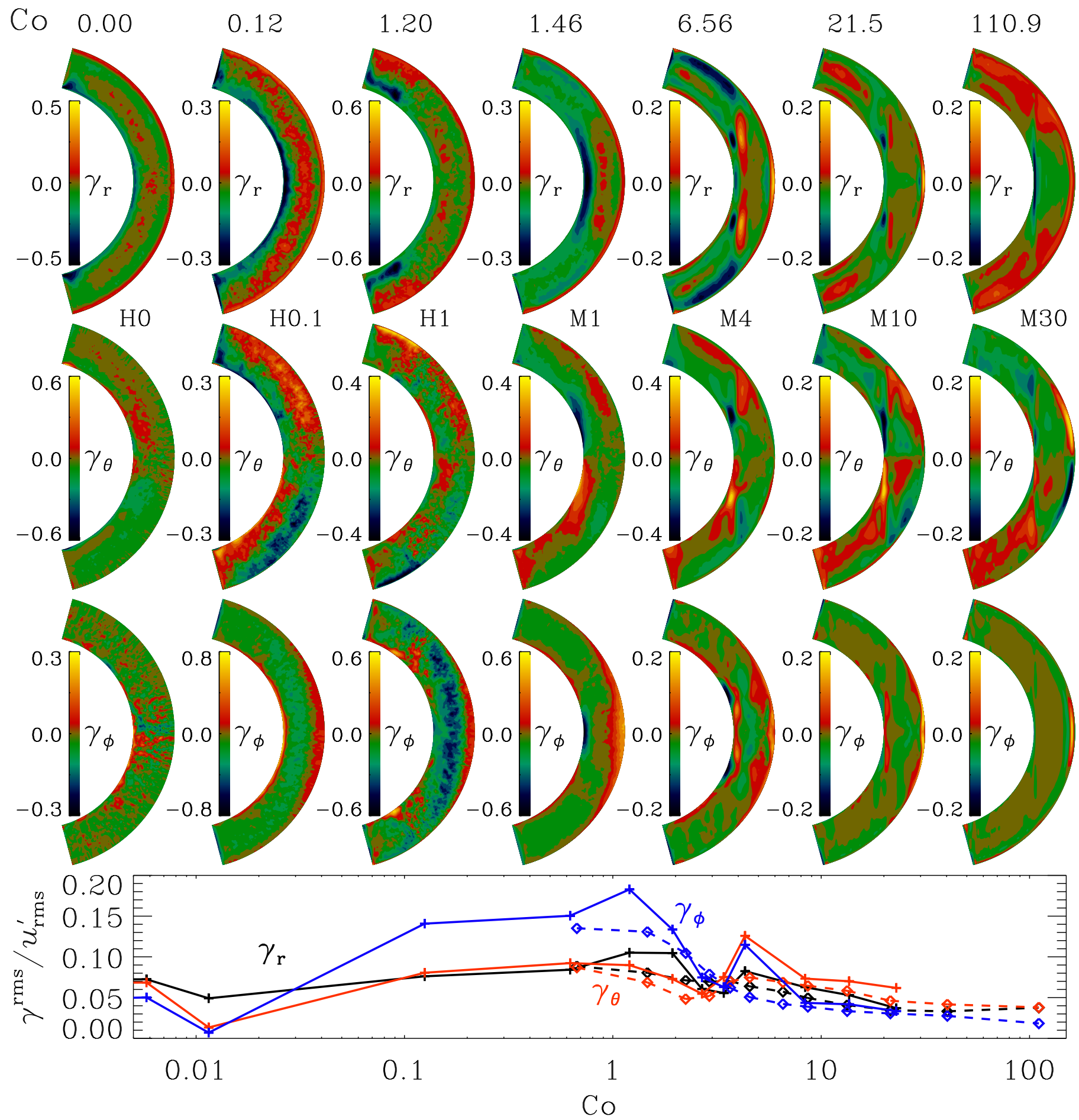

Fig. 7. Rotational dependency of the $\gamma$ components. We show meridional profiles of $\gamma_{r}$ (top row), $\gamma_{\theta}$ (second row), and $\gamma_{\phi}$ (third row), and their rms values (bottom row), with $\gamma_{r}$ (black lines), $\gamma_{\theta}$ (red), and $\gamma_{\phi}$ (blue). As in Fig. 3, the values of the HD runs (Set H) are shown with a solid line and crosses, whereas the MHD runs (Set M) are shown with a dashed line with diamonds. All values are normalized by $u_{\mathrm{rms}}^{\prime}$. The zero rotation run has been moved to $\mathrm{Co}=10^{-4}$ to be visible in the lower panel.

dominates the moderately rotating runs ( $\mathrm{Co}=1$ to 11$), \alpha_{\phi \phi}$ dominates the rapidly rotating ones. For the highest rotation rates, the peak values are three to four times larger and the rms values are even five to eight times larger than for $\alpha_{r r}$ and $\alpha_{\theta \theta}$.

All in all, the $\alpha$ tensor is highly anisotropic for all rotation rates above $\mathrm{Co} \approx 1$. The nature of the anisotropy changes from the moderate dominance of $\alpha_{r r}$ to the strong dominance of $\alpha_{\phi \phi}$ for $\mathrm{Co}>10$. For rapidly rotating stars, spherical coordinates are not optimal and the anisotropy of the $\alpha$ tensor is even more easily seen if we remap it to cylindrical coordinates $(\rho, \phi, z)$. Then, as shown in Fig. A.3, $\alpha_{z z}$ becomes close to zero. This is in agreement with theoretical predictions (Ruediger 1978; Krause \& Rädler 1980) and also roughly agrees with the axi- to nonaxisymmetric dynamo solution transition found by Viviani et al. (2018; their limiting Co having been around 3). However, the latter study did not report any quantitative change of the dynamo solutions at higher rotation 
J. Warnecke and M. J. Käpylä: Rotational dependence of turbulent transport coefficients
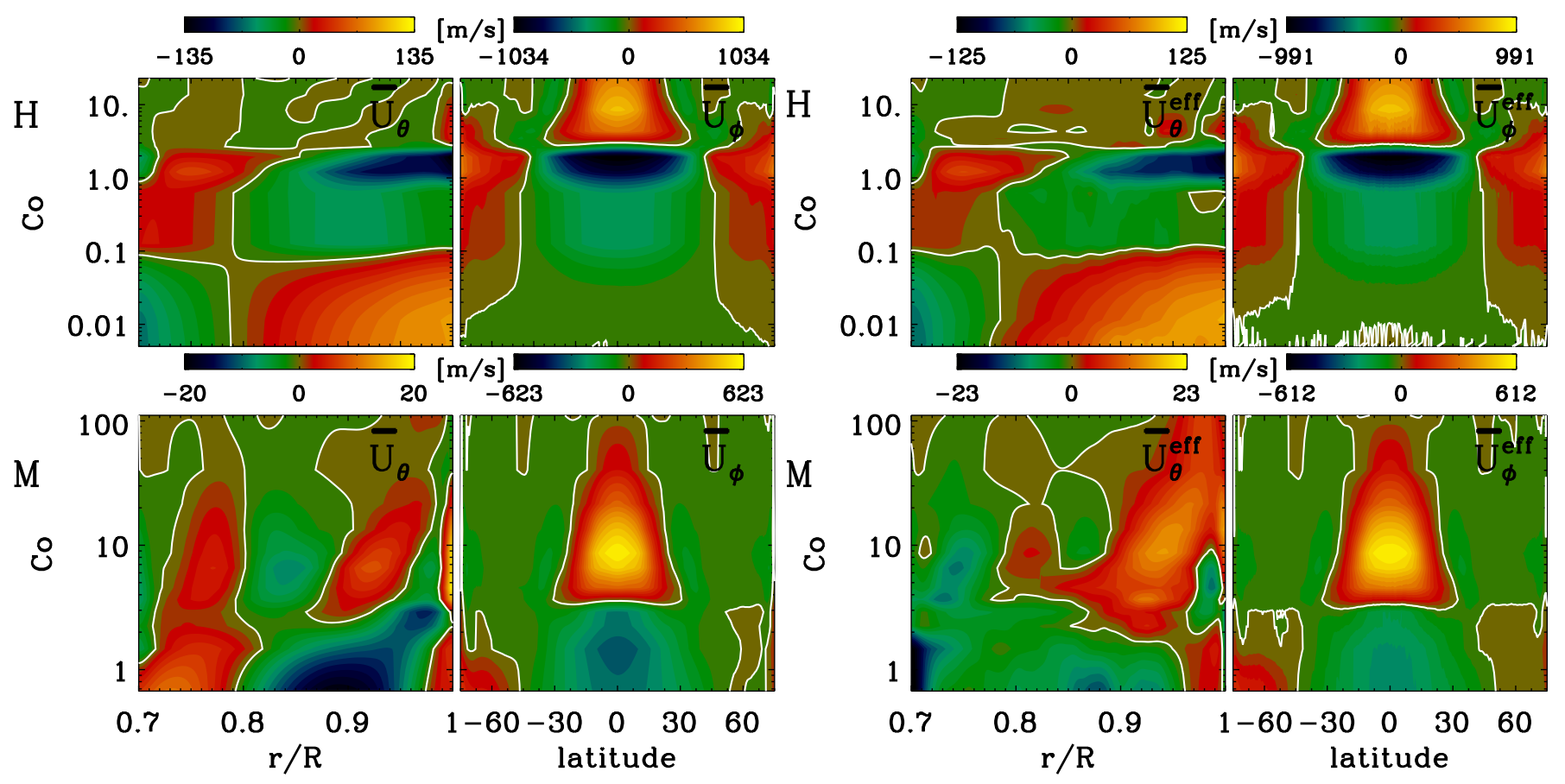

Fig. 8. Rotational dependency of meridional circulation and differential rotation and their effective counterparts. We plot the meridional circulation $\bar{U}_{\theta}$ (first column) and $\bar{U}_{\theta}^{\text {eff }}$ (third column) at $25^{\circ}$ latitude together with the azimuthal mean velocity $\bar{U}_{\phi}$ (second column) and $\bar{U}_{\phi}^{\text {eff }}$ (fourth column) close to the surface at $r=0.99 R$ as a function Co for the HD runs (Set H) in the top row and for the MHD runs (Set M) in the bottom row. Positive(negative) values of $\bar{U}_{\theta}$ are equatorward (poleward) and positive (negative) $\bar{U}_{\phi}$ are prograde(retrograde). The zero values are indicated with a white line.

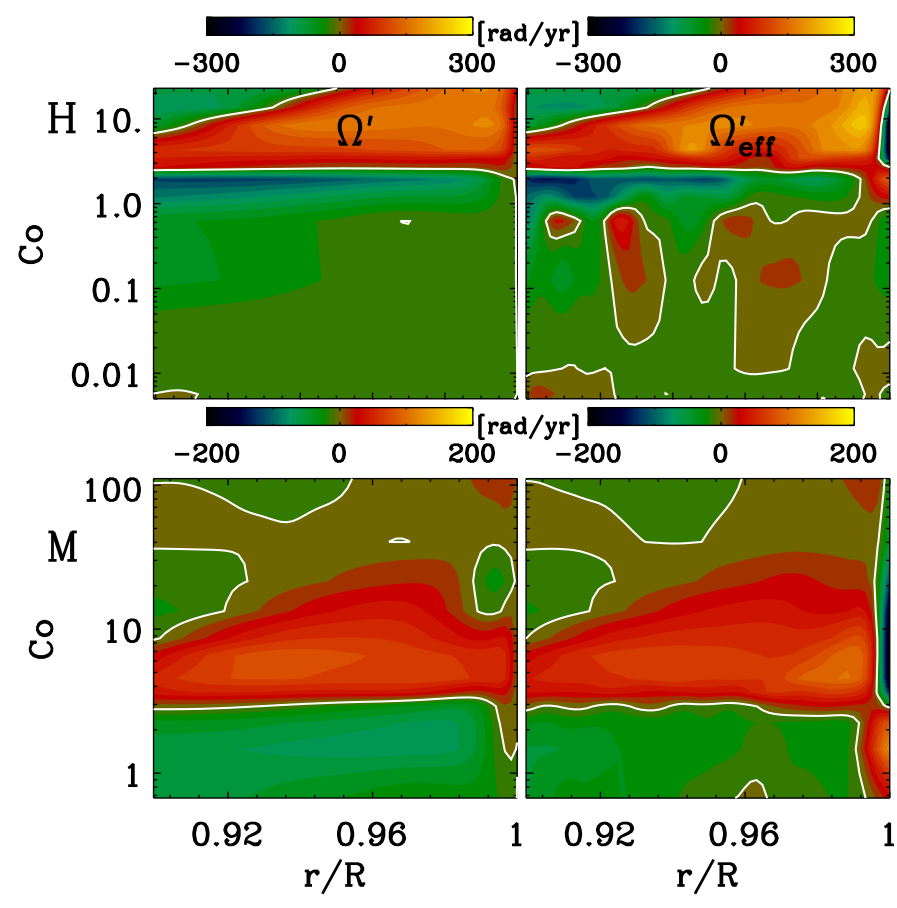

Fig. 9. Variation of radial shear of the mean flow $\Omega^{\prime}$ and the effective flow $\Omega_{\text {eff }}^{\prime}$ with rotation. We plot $\Omega^{\prime}$ (first column) and $\Omega_{\text {eff }}^{\prime}$ (second column) at $25^{\circ}$ latitude as a function of Co for the HD runs (Set $\mathrm{H}$ ) in the top row and for the MHD runs (Set M) in the bottom row. The zero values are indicated with a white line.

rates, where according to our results the anisotropies should play the most important role. In any case, it seems that anisotropies in the $\alpha$ effect might play some role in the generation of the nonax- isymmetric modes. This coexistence confirms the mean-field calculation (e.g., Rädler et al. 1990; Elstner \& Rüdiger 2007; Pipin 2017), where an anisotropic $\alpha$ tensor can generate nonaxisymmetric fields.

\subsection{Turbulent pumping}

Now, we investigate the rotational influence of the turbulent pumping vector $\gamma$ : see Fig. 7. All of its components have a much weaker dependence on rotation than those of $\alpha$. The rms value of $\gamma_{r}$ increases slightly with rotation up to $\mathrm{Co}=2$ and then decreases for higher rotation. For all runs, we find an upward pumping near the surface, agreeing with previous studies (Warnecke et al. 2018; Viviani et al. 2019). However, some changes of the spatial profile can be distinguished as increasing rotation: For slow rotation, we find the tendency for upward pumping in the bulk of the convection zone and downward pumping near the bottom. For $\mathrm{Co}=6.6, \gamma_{r}$ is also pointing downward at high latitudes. Furthermore, $\gamma_{\theta}$ also does not depend strongly on rotation. The HD runs exhibit some non-monotonic behavior in the form of an abrupt increase at around $\mathrm{Co}=6.6$, but this bump is absent in the MHD runs. For all runs with rotation, the spatial distribution shows equatorward pumping near the surface of the upper part of the convection zone and poleward pumping near the bottom. $\gamma_{\phi}$ shows the strongest rotational dependency of all the turbulent pumping coefficients. For rotation rates up to $\mathrm{Co}=1.2, \gamma_{\phi}$ increases and for higher $\mathrm{Co}$ it decreases, in particular in the MHD cases. Again, the HD cases show non-monotonic behavior at the same rotation rate as $\gamma_{\theta}$. The spatial structure seems to be mostly independent of rotation. For all runs with rotation, the pumping is prograde near the surface and at the bottom of the convection zone, and weakly retrograde in the bulk of the convection zone. All values of $\gamma$ are 

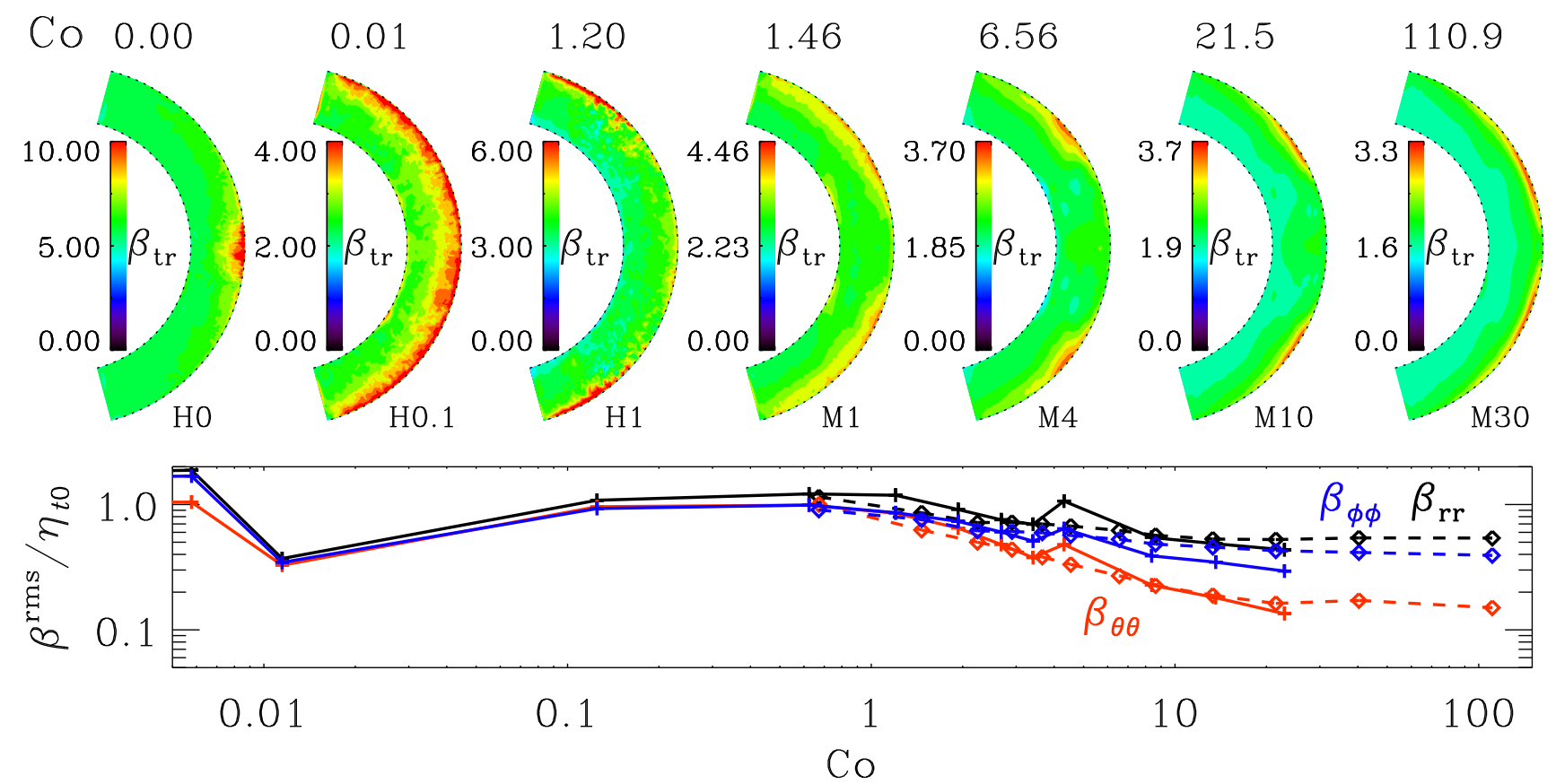

Fig. 10. Rotational dependency of the turbulent diffusion as the trace of $\beta$. We show meridional profiles of $\beta_{\text {tr }}$ (top row) and the rms values of the diagonal component (bottom row), with $\beta_{r r}$ (black lines), $\beta_{\theta \theta}$ (red), and $\beta_{\phi \phi}$ (blue). As in Fig. 3, the values of the HD runs (Set H) are shown with a solid line and crosses, whereas the MHD runs (Set M) are shown with a dashed line with diamonds. All values are normalized by $\eta_{\mathrm{t} 0}=\tau_{\mathrm{tur}} u_{\mathrm{rms}}^{\prime 2} / 3$. The zero rotation run has been moved to $\mathrm{Co}=10^{-4}$ to be visible in the lower panel.

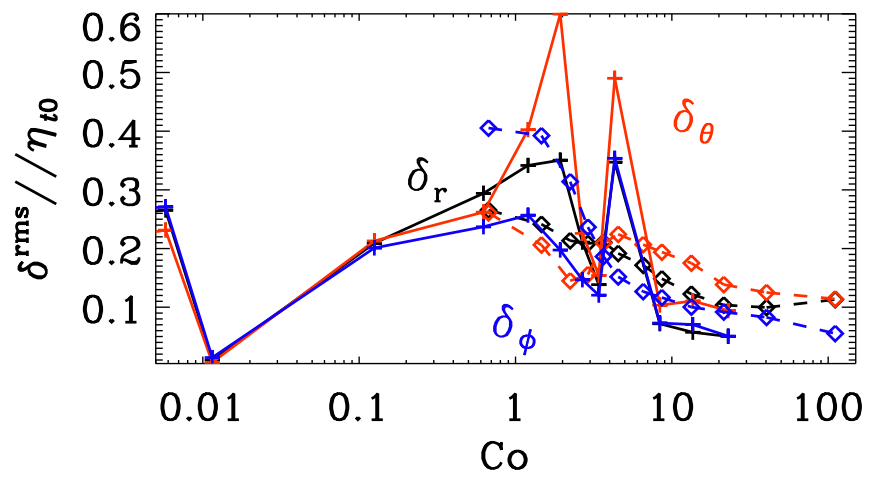

Fig. 11. Rotational dependency of the Rädler effect. We show the rms values of the components of $\boldsymbol{\delta}$ with $\delta_{r}$ (black lines), $\delta_{\theta}$ (red), and $\delta_{\phi}$ (blue). As in Fig. 3, the values of the HD runs (Set $\mathrm{H}$ ) are shown with a solid line and crosses, whereas the MHD runs (Set M) are shown with a dashed line with diamonds. All values are normalized by $\eta_{\mathrm{t} 0}=$ $\tau_{\text {tur }} u_{\mathrm{rms}}^{\prime 2} / 3$. The zero rotation run has been moved to Co $=10^{-4}$ to be visible.

weaker than the turbulent velocity $u_{\mathrm{rms}}^{\prime}$; for most cases they are only around 10 to $20 \%$ of $u_{\mathrm{rms}}^{\prime}$.

To investigate how the turbulent pumping influences the evolution of the magnetic field, we calculate the effective velocity, $\overline{\boldsymbol{U}}^{\text {eff }}=\overline{\boldsymbol{U}}+\gamma$, which the magnetic field is sensitive to. As shown in previous studies (Warnecke et al. 2018; Viviani et al. 2019), $\boldsymbol{\gamma}$ can have a large impact on $\overline{\boldsymbol{U}}^{\text {eff }}$. To this end, Fig. 8 shows the effective meridional and azimuthal flow (differential rotation) together with the original meridional and azimuthal flow. The azimuthal turbulent pumping is too weak to significantly alter $\bar{U}_{\phi}^{\text {eff }}$. However, we find that the anti-solar differential rotation in the MHD runs is weaker due to $\gamma_{\phi}$. For the HD runs, $\bar{U}_{\theta}^{\mathrm{eff}}$ only changes for the solar-like differential rotation. There, the multi-cellular structure is altered to a noncellular structure. For the MHD runs, the influence of $\gamma_{\theta}$ on $\bar{U}_{\theta}^{\text {eff }}$ is drastic. The equatorward flow in the lower part of the convection zone completely vanishes and even becomes equatorward for some rotation rates. Moreover, the other flow structures are significantly altered.

Even though we do not find strong changes in $\bar{U}_{\phi}^{\text {eff }}$ due to $\gamma_{\phi}$, the radial shear can nevertheless change (Warnecke et al. 2018). To check for this possible effect, we plot in Fig. 9 the radial shear defined as $\Omega^{\prime}=r \sin \theta \partial \Omega / \partial r$ and $\Omega_{\text {eff }}^{\prime}=r \sin \theta \partial \Omega^{\text {eff }} / \partial r$ with $\Omega^{\text {eff }}=\bar{U}_{\phi}^{\text {eff }} / r \sin \theta+\Omega_{0}$. For weak rotation, we find that the dominantly negative shear changes to positive values in the bulk of the convection zone for slow to moderate rotation rates. More dramatic are the changes near the surface. For nearly all runs, an additional layer of shear of opposite sign to the overall shear in the bulk is generated near the very surface. For slow rotation rates, a positive shear region appears, while for high rotation, a negative one appears. Hence, our results support the conclusion of Warnecke et al. (2018) and Viviani et al. (2019), namely that the turbulent pumping plays an important role for the evolution of the magnetic field.

\subsection{Turbulent diffusion and the Rädler effect}

To investigate the rotational dependency of turbulent diffusion, we limit ourselves to the diagonal components and the trace of $\beta, \beta_{\text {tr }}=\beta_{r r}+\beta_{\theta \theta}+\beta_{\phi \phi}$. As shown in Fig. $10, \beta_{\text {tr }}$ decreases with rotation and we find that it is often two times stronger near the surface than in the lower part of the convection zone. The trace is always larger than zero, with the exception of some values at the radial and latitudinal boundaries, which might be artifacts. The rms values of the diagonal components remain roughly independent of rotation until $\mathrm{Co}=7$. In this regime, all components have strengths close to $\eta_{\mathrm{t} 0}$. For higher rotation, all three diagonal components decrease with rotation up to $\mathrm{Co}=22$, the decrease being most pronounced for the $\theta \theta$ component whose values become 


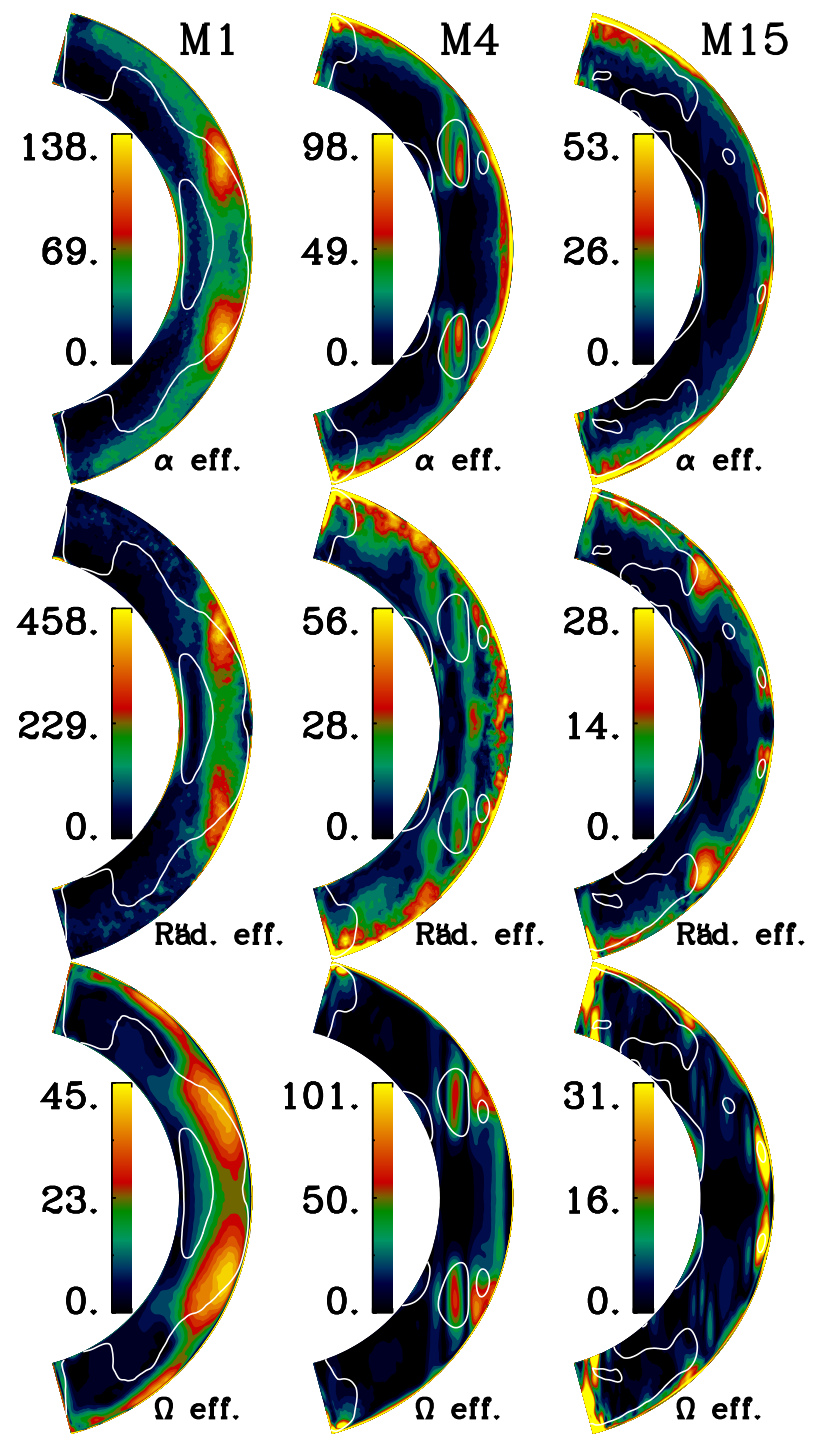

Fig. 12. Dynamo effects generating $\bar{B}_{\phi}$ for Runs M1, M4, and M15. We show the $\alpha$ effect (top row), the Rädler/ $\delta$ effect (middle), and the $\Omega$ effect (bottom), where we overplot as white contours the rms values of $\bar{B}_{\phi}$ above half of the maximum, indicating the magnetic field region used in the calculation of the dynamo effects shown in Fig. 13. All values are given in $\mathrm{kG} \mathrm{yr}^{-1}$.

roughly four times smaller than $\beta_{r r}$ and three times smaller than $\beta_{\phi \phi}$. For even higher rotation, the $\beta$ components remain roughly constant. As for the $\alpha$ tensor, we also map the $\beta$ tensor to cylindrical coordinates and plot its diagonal components in Fig. A.4. The differences between the presentations in the two coordinate systems are not large. Now $\beta_{z z}$ is the weakest of all three diagonal components, whereas $\beta_{\rho \rho}$ and $\beta_{\phi \phi}$ have similar values. This is in agreement with the theoretical prediction, according to which $\beta_{z z}$ should be most highly quenched for rapid rotation (Krause \& Rädler 1980).

Now, we turn to the Rädler effect, expressed by $\delta$. As shown in Fig. 11, all components of $\boldsymbol{\delta}$ increase for slow and decrease for fast rotation. For HD runs, $\delta_{\theta}$ becomes strong for the runs with a strong energy in differential rotation $E_{\mathrm{kin}}^{\mathrm{dif}}$ : in the anti-solar regime, Run H1.5, and in the solar-like regime, Run H3. Furthermore, $\delta_{r}$ is also strong in the former run and $\delta_{\phi}$ in the latter. For Co $>4, \delta_{\theta}$ is dominating the other components in both HD and MHD runs. The values of all $\delta$ components are of the order of $10-30 \%$ of $\eta_{\mathrm{t} 0}$.

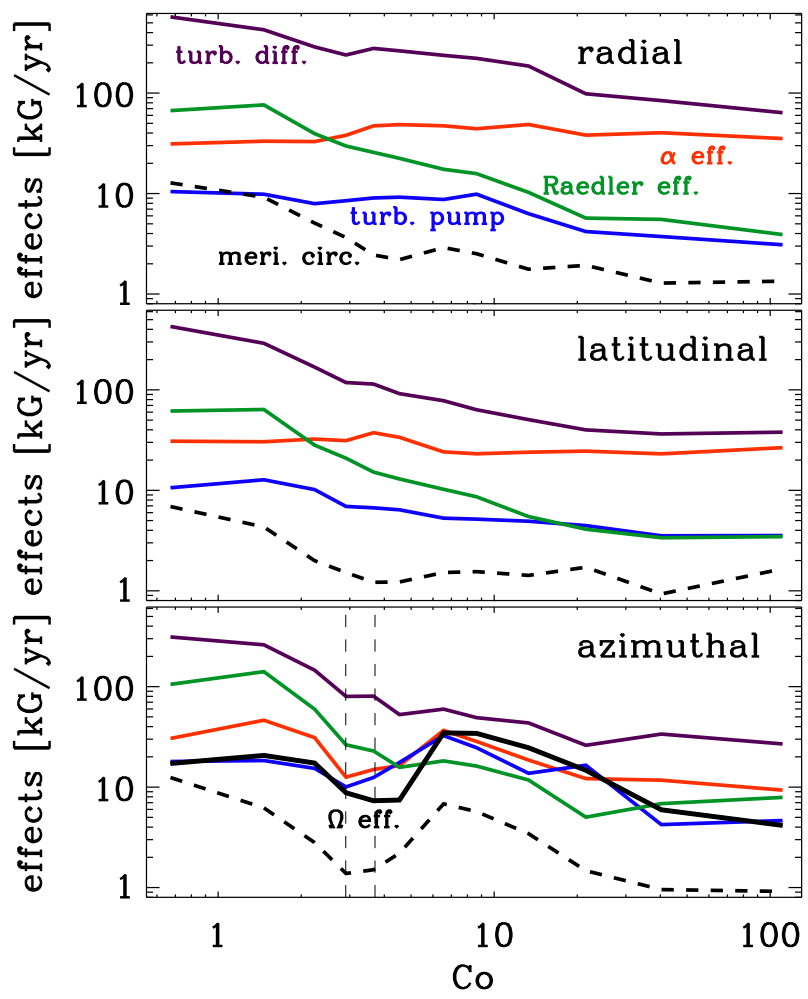

Fig. 13. Rotational dependence of the main dynamo effects related to the radial field (top panel), the latitudinal field (middle), and the azimuthal field evolution (bottom) with the $\alpha$ effect (red), the turbulent diffusion (purple), the turbulent pumping (blue), the meridional circulation (black dashed), the Rädler effect (green), and the $\Omega$ effect (black solid). The vertical dashed lines indicate the transition from the anti-solar to solarlike differential rotation. See Sect. 3.7 for the calculation details.

\subsection{Rotational dependency of the dynamo mechanism}

To test which dynamo effects are responsible for the generation and evolution of the magnetic field, we individually monitor the following terms in the induction equation:

$\boldsymbol{\nabla} \times \boldsymbol{\alpha} \cdot \overline{\boldsymbol{B}} \quad \alpha$ effect,

$\boldsymbol{\nabla} \times \boldsymbol{\gamma} \times \overline{\boldsymbol{B}}$ turbulent pumping,

$\boldsymbol{\nabla} \times \boldsymbol{\beta} \cdot \boldsymbol{\nabla} \times \overline{\boldsymbol{B}}$ turbulent diffusion,

$\boldsymbol{\nabla} \times \boldsymbol{\delta} \times \boldsymbol{\nabla} \times \overline{\boldsymbol{B}}$ Rädler effect,

$\boldsymbol{\nabla} \times\left(\overline{\boldsymbol{u}}_{\text {dif }} \times \overline{\boldsymbol{B}}\right) \quad \Omega$ effect,

$\boldsymbol{\nabla} \times\left(\overline{\boldsymbol{u}}_{\mathrm{mer}} \times \overline{\boldsymbol{B}}\right) \quad$ meridional circulation,

where $\overline{\boldsymbol{u}}_{\text {dif }}=\left(0,0, \overline{u_{\phi}}\right)$ and $\overline{\boldsymbol{u}}_{\text {mer }}=\left(\overline{u_{r}}, \overline{u_{\theta}}, 0\right)$. We then take the temporal rms $\sqrt{\left\langle\cdot{ }^{2}\right\rangle_{t}}$ of each of these terms. In Fig. 12, we show an example of the field generators for $\bar{B}_{\phi}$ from the $\alpha$, Rädler, and $\Omega$ effects from three different runs. We see for Run M1 that the strongest effect is the Rädler effect, which acts on the same location as the $\alpha$ effect. The mean azimuthal field, the strongest field regions being indicated with white contour lines, actually concentrates closer to the equator and nearer to the bottom of the convection zone than the distribution of the field generators. This can be explained by the meridional pumping which is equatorward at these locations, added with downwards-directed $\gamma_{r}$ in the bottom parts of the convection zone. For M4 and M10 with 
$\mathrm{Co}=6.6$ and 22, oscillatory magnetic fields with clear equatorward migration are excited, as can be seen from Fig. A.5. From Fig. 12 we see that the $\Omega$ and the $\alpha$ effects are strong in the areas where the azimuthal field is also strong. For even higher rotation in Run M15, the field generation near the surface near the equator is mostly due to the $\alpha$ effect with a contribution from the $\delta$ and $\Omega$ effects. For the high-latitude fields, $\alpha$ and Rädler effects have stronger contributions. The magnetic field evolution shows an irregular solution with an indication of poleward migrating field which might fit to this kind of dynamo. This result also shows that even if the $\alpha$ tensor is highly anisotropic with very low values for $\alpha_{r r}$ and $\alpha_{\theta \theta}$, the $\alpha$ effect generating the azimuthal field is still strong and can sustain a strong large-scale dynamo.

To further refine our analysis, we adopt the approach by Warnecke (2018) to measure the dynamo effects only from the locations where the magnetic field component that they are acting on is larger than the half maximum of its rms value (indicated by white contour lines in Fig. 12). We average each dynamo effect over these locations and plot all of them as a function of rotation in Fig. 13.

From Fig. 13 we find that the dynamo effects show a strong rotational dependence. The effect of turbulent diffusion decreases for all runs for increasing rotation, even though the diagonal components of $\beta$ become constant. This is most likely due to the decrease of turbulent intensity with rotation, which is used as a normalization in Fig. 10. Except for slow rotation $(\mathrm{Co}=0.7-1.5)$, the effect of the turbulent pumping is significantly stronger than the effect on the meridional circulation. Both effects decrease with increasing rotation.

For the other effects, we find three distinct regimes. The first regime is for slow rotation, where the differential rotation is antisolar. There, the Rädler and the $\alpha$ effects are dominating even over the $\Omega$ effect for azimuthal field generation. This would rule out an $\alpha \Omega$ effect and suggest an $\alpha^{2}$-type dynamo with a strong $\delta$ contribution. From our method of using averaged rms values without signs, we cannot conclude whether the Rädler effect contributes to the magnetic field enhancement or the diffusion of the field, but nevertheless our analysis indicates a strong role of this effect in the evolution of the magnetic field. This is consistent with the finding of Viviani et al. (2019), where the authors find indications for an $\alpha^{2}$-type model with a strong $\delta$ contribution in their run with $\mathrm{Co}=2.8$. However, these latter authors find a cyclic magnetic field solution in contrast to our stationary or irregular ones, as shown in Fig. A.5. For the runs in the transitionary phase of the differential rotation (Runs M2 to M3, with $\mathrm{Co}=2.9$ to 4.5 ), the $\Omega$ effect is even weaker than for slow rotation, and the dynamo is dominated by an $\alpha^{2}$ contribution with a much weaker $\delta$. However, we see clearer indication of cycles, which are nevertheless not yet very pronounced; see Fig. A.5.

The second regime is one where the runs exhibit solar-like differential rotation profiles. There, the $\Omega$ effect is comparable to or even dominates the $\alpha$ effect in generating the azimuthal field. Runs in this regime (M4 to M10 with $\mathrm{Co}=6.6$ to 22) show clear equatorward migration of the oscillating magnetic fields. Previous analyses of similar runs have revealed that these dynamo waves can be explained by the Parker-Yoshimura rule (Warnecke et al. 2018; Warnecke 2018) for $\alpha \Omega$ dynamos. This is consistent with our finding of the $\Omega$ effect being large in Fig. 13. The strong contribution that we find for $\alpha$ in generating the azimuthal field can still contribute to the magnetic field evolution, but it does not necessarily influence the period and propagation direction of the dynamo wave, as was the case also in the studies of Warnecke et al. (2018) and Warnecke (2018).

The third regime is observed for runs with the highest rotation rates $(\mathrm{M} 15 \& \mathrm{M} 30$, with $\mathrm{Co}=40 \& 111)$. The Rädler effect has decreased with rotation for the poloidal components of the field, while the $\alpha$ effect has remained roughly constant. For the azimuthal component, the $\Omega$ effect drops to much lower values, as already indicated by the decrease of the overall energy in the differential rotation in Fig. 1. The Rädler effect for this component is comparable to the $\alpha$ effect. This indicates an $\alpha^{2}$ dynamo with a weak $\delta$ contribution for the azimuthal magnetic field.

With the rotational dependence of the dynamo effects, we can now understand why the ratio of magnetic to kinetic energy increases even though the shear and normalized $\boldsymbol{\alpha}$ tensor remain constant for moderate to high rotation. This is because simultaneously turbulent diffusion decreases as a function of rotation, enabling a more efficient dynamo action. This is in very good agreement with the results of Käpylä et al. (2009), who find similar behavior from turbulent convection in Cartesian domains where rotational influence was varied.

\section{Conclusions}

We performed a comprehensive study of how turbulent transport coefficients measured from global convective dynamo simulations depend on rotation in solar-like stars. For this, we varied the rotational influence of convection in terms of Coriolis number from $\mathrm{Co}=0$ to $\mathrm{Co}=110$. We found that the normalized trace of $\alpha$ only increases up to Co $=4$, with an approximate power law of $\mathrm{Co}^{0.5}$, and then levels off. The trace of $\boldsymbol{\alpha}$ shows a very similar spatial profile in comparison to an expression of $\alpha$ based on the kinetic helicity, $\alpha_{\mathrm{K}}$. However, this quantity does not level off, but continues its growth even in the rapid rotation regime. However, if we take into account that the length scales of convection become reduced with increasing rotational influence, the effect of which could result in a decrease of the correlation time with a power law of $\mathrm{Co}^{-0.5}$ according to theoretical considerations (Chandrasekhar 1961) and recent numerical results (Featherstone \& Hindman 2016; Viviani et al. 2018), then $\alpha_{\mathrm{K}}$ also levels off. The magnetic correction to the $\alpha$ effect, expressed in terms of $\alpha_{\mathrm{M}}$ based on the current helicity, becomes anomalously strong in the cases of rapid rotation, even exceeding the value of $\alpha_{\mathrm{K}}$ locally. Therefore, it is not justified to consider $\alpha_{\mathrm{M}}$ as a perturbation, as in the original analysis of Pouquet et al. (1976). However, we treated the turbulent correlation times for the flow and magnetic fields equally in our analysis, which might explain the discrepancy. This issue requires further investigation, but even at this stage it is clear that some caution is needed when deriving the turbulent transport coefficients from convection simulations using these proxies.

We further find that the $\boldsymbol{\alpha}$ tensor becomes highly anisotropic for Co $>1$, as expected from theoretical predictions (Krause \& Rädler 1980). In the moderate rotation regime, $\alpha_{r r}$ dominates over the other diagonal components. The nature of the anisotropy changes for $\mathrm{Co}>10$, when $\alpha_{r r}$ and $\alpha_{\theta \theta}$ become strongly reduced, while $\alpha_{\phi \phi}$ strongly increases. Anisotropies in the $\boldsymbol{\alpha}$ tensor are one of the candidates leading to nonaxisymmetric large-scale dynamo solutions (e.g., Rädler et al. 1990; Elstner \& Rüdiger 2007; Pipin 2017). However, the transition to nonaxisymmetry was seen at somewhat elevated Coriolis numbers of roughly three in the study of Viviani et al. (2018), where similar runs to those here were reported, but with the full longitudinal extent and therefore capable of naturally exciting nonaxisymmetric large-scale modes. The difficulty in analyzing such runs with the test-field method arises from the fact that the axisymmetric averages are not suitable for nonaxisymmetric dynamo solutions. Confirmation of the importance of $\alpha$ effect anisotropies in the excitation of nonaxisymmetric modes must therefore await further development of the test-field method 
and/or appropriate 3D mean-field modeling taking into account the dynamo effects measured here.

The turbulent pumping components do not strongly depend on rotation. In all runs, we measure upward pumping near the surface, in contrast to what is needed for the surface-flux-transport models to agree with solar observations (e.g., Cameron et al. 2012). The latitudinal pumping is mostly equatorward in the upper part of the convection zone and poleward in the lower part, and therefore it could be able to advect a dynamo wave equatorward if it overcame diffusion. However, this is not seen in any of our models. The presence of the latitudinal pumping completely alters the effective meridional circulation, which has strong implications for flux-transport dynamo models that rely on certain types of meridional circulation profiles and do not fully consider all the turbulent effects. The azimuthal pumping leads to a sharp sign change for the effective shear near the surface for all runs.

We find that the normalized turbulent diffusion decreases slightly with rotation before it levels off at around Co $>10$ and becomes weakly anisotropic. $\delta$, describing the Rädler effect, is the strongest for moderate rotation, where differential rotation is also the strongest.

Analyzing the dynamo effects as a function of rotation reveals three distinct regimes. For slow rotation, we find strong $\alpha$ and Rädler effects together with anti-solar differential rotation, consistent with the work of Viviani et al. (2019). For moderate rotation, where differential rotation is solar-like and the magnetic field develops equatorward migration with clearly defined cycles, we find a strong contribution from both $\alpha$ and $\Omega$ effects for the generation of the toroidal component and from the $\alpha$ effect for the generation of the poloidal component, while the other effects remain subdominant. This is consistent with an $\alpha \Omega$ or $\alpha^{2} \Omega$ dynamo, in agreement with previous studies (Warnecke et al. 2018; Warnecke 2018). For high rotation, the $\alpha$ effect contributions remain strong, while the $\Omega$ effect is significantly reduced. Therefore, our interpretation is that a dynamo of $\alpha^{2}$ type, with some influence of the Rädler effect for azimuthal field, is operating in this regime. The dynamo efficiency, defined as the ratio of magnetic energy to kinetic energy, increases linearly with $\mathrm{Co}$, in agreement with previous studies (Viviani et al. 2018; Augustson et al. 2019). This can be explained by the $\alpha$ effect being almost independent of rotation, whereas the effect of turbulent diffusion decreases with rotation in agreement with the Cartesian models of Käpylä et al. (2009).

Acknowledgements. We thank the anonymous referee and our colleague Petri J. Käpylä for constructive comments on the manuscript. The simulations have been carried out on supercomputers at GWDG, on the Max Planck supercomputer at RZG in Garching and in the facilities hosted by the CSC-IT Center for Science in Espoo, Finland, which are financed by the Finnish ministry of education. J. W. acknowledges funding by the Max-Planck/Princeton Center for Plasma Physics and from the People Programme (Marie Curie Actions) of the European Union's Seventh Framework Programme (FP7/2007-2013) under REA Grant agreement No. 623609. This project has received funding from the European Research Council (ERC) under the European Union's Horizon 2020 research and innovation programme (grant agreement n:o 818665 "UniSDyn") and has been supported from the Academy of Finland Centre of Excellence ReSoLVE (project number 307411).

\section{References}

Augustson, K., Brun, A. S., Miesch, M., \& Toomre, J. 2015, ApJ, 809, 149 Augustson, K. C., Brun, A. S., \& Toomre, J. 2019, ApJ, 876, 83

Barekat, A., Schou, J., \& Gizon, L. 2014, A\&A, 570, L12

Beaudoin, P., Simard, C., Cossette, J.-F., \& Charbonneau, P. 2016, ApJ, 826, 138 Boro Saikia, S., Marvin, C. J., Jeffers, S. V., et al. 2018, A\&A, 616, A108

Brandenburg, A., \& Giampapa, M. S. 2018, ApJ, 855, L22

Brandenburg, A., \& Subramanian, K. 2005, Phys. Rep., 417, 1
Brandenburg, A., Saar, S. H., \& Turpin, C. R. 1998, ApJ, 498, L51

Brandenburg, A., Rädler, K.-H., Rheinhardt, M., \& Subramanian, K. 2008, ApJ, 687, L49

Cameron, R. H., Schmitt, D., Jiang, J., \& Ișıı, E. 2012, A\&A, 542, A127

Chandrasekhar, S. 1961, Hydrodynamic and Hydromagnetic Stability (Oxford: Clarendon)

Cole, E., Käpylä, P. J., Mantere, M. J., \& Brandenburg, A. 2014, ApJ, 780, L22

Duarte, L. D. V., Wicht, J., Browning, M. K., \& Gastine, T. 2016, MNRAS, 456 , 1708

Elstner, D., \& Rüdiger, G. 2007, Astron. Nachr., 328, 1130

Fan, Y., \& Fang, F. 2014, ApJ, 789, 35

Featherstone, N. A., \& Hindman, B. W. 2016, ApJ, 818, 32

Featherstone, N. A., \& Miesch, M. S. 2015, ApJ, 804, 67

Gastine, T., Yadav, R. K., Morin, J., Reiners, A., \& Wicht, J. 2014, MNRAS, 438, L76

Ghizaru, M., Charbonneau, P., \& Smolarkiewicz, P. K. 2010, ApJ, 715, L133

Käpylä, P. J., Korpi, M. J., \& Brandenburg, A. 2009, A\&A, 500, 633

Käpylä, P. J., Mantere, M. J., \& Brandenburg, A. 2012, ApJ, 755, L22

Käpylä, P. J., Mantere, M. J., Cole, E., Warnecke, J., \& Brandenburg, A. 2013, ApJ, 778, 41

Käpylä, P. J., Käpylä, M. J., \& Brandenburg, A. 2014, A\&A, 570, A43

Käpylä, M. J., Käpylä, P. J., Olspert, N., et al. 2016, A\&A, 589, A56

Käpylä, P. J., Käpylä, M. J., Olspert, N., Warnecke, J., \& Brandenburg, A. 2017, A\&A, 599, A4

Karak, B. B., Käpylä, P. J., Käpylä, M. J., et al. 2015, A\&A, 576, A26

Kitchatinov, L. L., \& Rüdiger, G. 1999, A\&A, 344, 911

Krause, F., \& Rädler, K.-H. 1980, Mean-field Magnetohydrodynamics and Dynamo Theory (Oxford: Pergamon Press)

Lehtinen, J., Jetsu, L., Hackman, T., Kajatkari, P., \& Henry, G. W. 2016, A\&A, 588, A38

Morin, J., Donati, J.-F., Petit, P., et al. 2010, MNRAS, 407, 2269

Moss, D., \& Brandenburg, A. 1995, Geophys. Astrophys. Fluid Dyn., 80, 229

Moss, D., Barker, D. M., Brandenburg, A., \& Tuominen, I. 1995, A\&A, 294, 155

Noyes, R. W., Hartmann, L. W., Baliunas, S. L., Duncan, D. K., \& Vaughan, A. H. 1984, ApJ, 279, 763

Olspert, N., Lehtinen, J. J., Käpylä, M. J., Pelt, J., \& Grigorievskiy, A. 2018, A\&A, 619, A6

Parker, E. N. 1955, ApJ, 122, 293

Pevtsov, A. A., Fisher, G. H., Acton, L. W., et al. 2003, ApJ, 598, 1387

Pipin, V. V. 2017, MNRAS, 466, 3007

Pizzolato, N., Maggio, A., Micela, G., Sciortino, S., \& Ventura, P. 2003, A\&A, 397, 147

Pouquet, A., Frisch, U., \& Léorat, J. 1976, J. Fluid Mech., 77, 321

Rädler, K.-H. 1969, Veröffentl. Geod. Geophys, 13, 131

Rädler, K.-H., Wiedemann, E., Brandenburg, A., Meinel, R., \& Tuominen, I. 1990, A\&A, 239, 413

Reiners, A., Schüssler, M., \& Passegger, V. M. 2014, ApJ, 794, 144

Reinhold, T., Reiners, A., \& Basri, G. 2013, A\&A, 560, A4

Ruediger, G. 1978, Astron. Nachr., 299, 217

Ruediger, G., \& Kichatinov, L. L. 1993, A\&A, 269, 581

Saar, S. H. 2001, in 11th Cambridge Workshop on Cool Stars, Stellar Systems and the Sun, eds. R. J. Garcia Lopez, R. Rebolo, \& M. R. Zapaterio Osorio, ASP Conf. Ser., 223, 292

Schrinner, M. 2011, A\&A, 533, A108

Schrinner, M., Rädler, K.-H., Schmitt, D., Rheinhardt, M., \& Christensen, U. 2005, Astron. Nachr., 326, 245

Schrinner, M., Rädler, K.-H., Schmitt, D., Rheinhardt, M., \& Christensen, U. R. 2007, Geophys. Astrophys. Fluid Dyn., 101, 81

Schrinner, M., Petitdemange, L., \& Dormy, E. 2011, A\&A, 530, A140

Schrinner, M., Petitdemange, L., \& Dormy, E. 2012, ApJ, 752, 121

Steenbeck, M., Krause, F., \& Rädler, K.-H. 1966, Z. Naturforsch. Teil A, 21, 369

Strugarek, A., Beaudoin, P., Charbonneau, P., Brun, A.S., \& do Nascimento, J.D. 2017, Science, 357,185

Thompson, M. J., Toomre, J., Anderson, E. R., et al. 1996, Science, 272, 1300

Tuominen, I., Berdyugina, S., \& Korpi, M. 2002, Astron. Nachr., 323, 367

Vidotto, A. A., Gregory, S. G., Jardine, M., et al. 2014, MNRAS, 441, 2361

Viviani, M., Warnecke, J., Käpylä, M. J., et al. 2018, A\&A, 616, A160

Viviani, M., Käpylä, M. J., Warnecke, J., Käpylä, P. J., \& Rheinhardt, M. 2019, ApJ, 886, 21

Warnecke, J. 2018, A\&A, 616, A72

Warnecke, J., \& Peter, H. 2019, A\&A, submitted, [arXiv:1910.06896]

Warnecke, J., Käpylä, P. J., Käpylä, M. J., \& Brandenburg, A. 2014, ApJ, 796, L12

Warnecke, J., Käpylä, P. J., Käpylä, M. J., \& Brandenburg, A. 2016, A\&A, 596, A115

Warnecke, J., Rheinhardt, M., Tuomisto, S., et al. 2018, A\&A, 609, A51

Wright, N. J., \& Drake, J. J. 2016, Nature, 535, 526

Yoshimura, H. 1975, ApJ, 201, 740 


\section{Appendix A: Rotation profiles and butterfly diagrams for all runs}

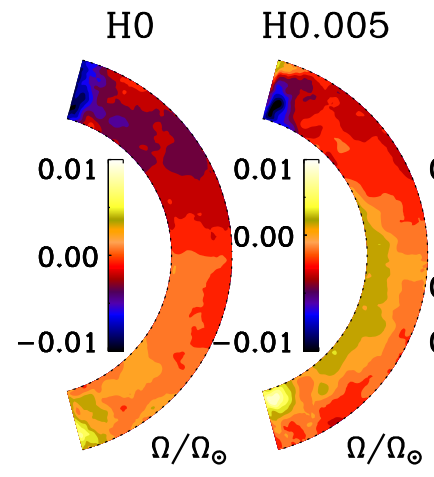

H0.1

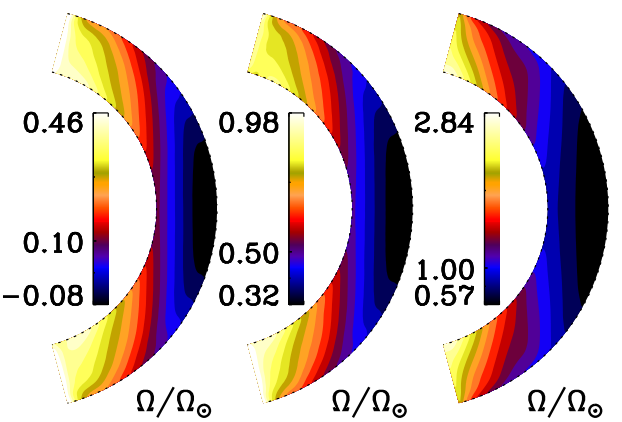

$\mathrm{H} 1.5$

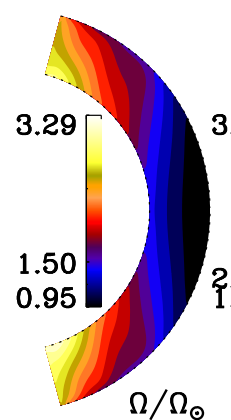

H2

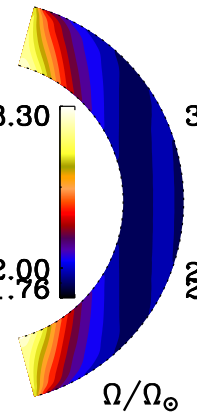

$\mathrm{H} 2.5$
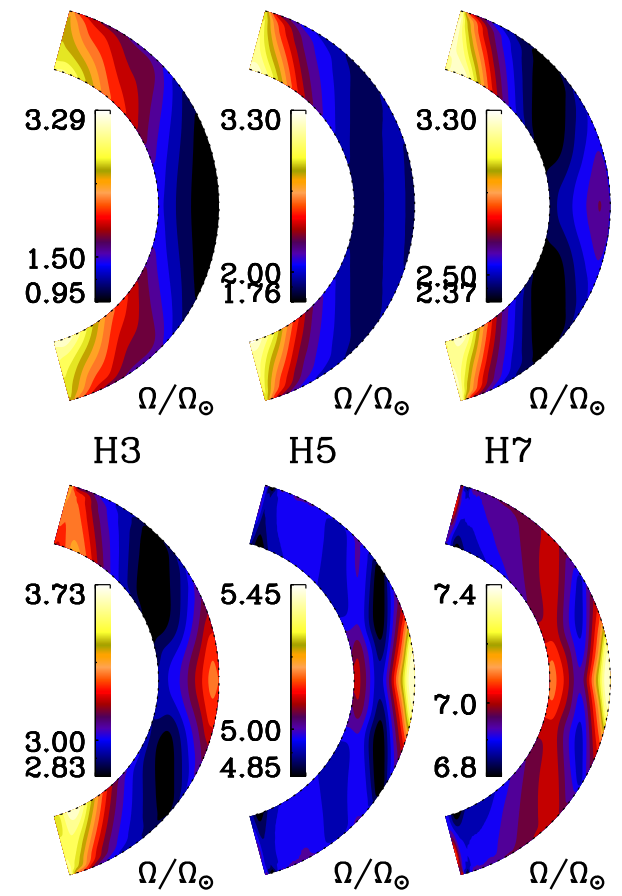

$\mathrm{H} 7$

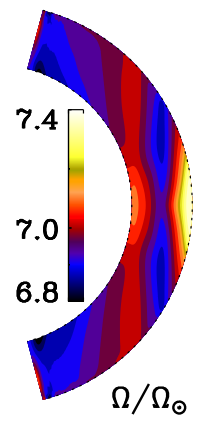

Fig. A.1. Normalized local rotation profile $\Omega / \Omega_{\odot}$ with $\Omega=\Omega_{0}+$ $\overline{\boldsymbol{u}} / r \sin \theta$ for all HD runs (Set $\mathrm{H}$ ), except run $\mathrm{H} 10$. The values of $\Omega$ have been calculated as a time average over the saturated state.

For completeness, in this Appendix we present the rotation profiles (Fig. A.1 for HD runs (Set H) and Fig. A.2 for MHD runs (Set M)) and butterfly diagrams (Fig. A.5) from all the runs ana-
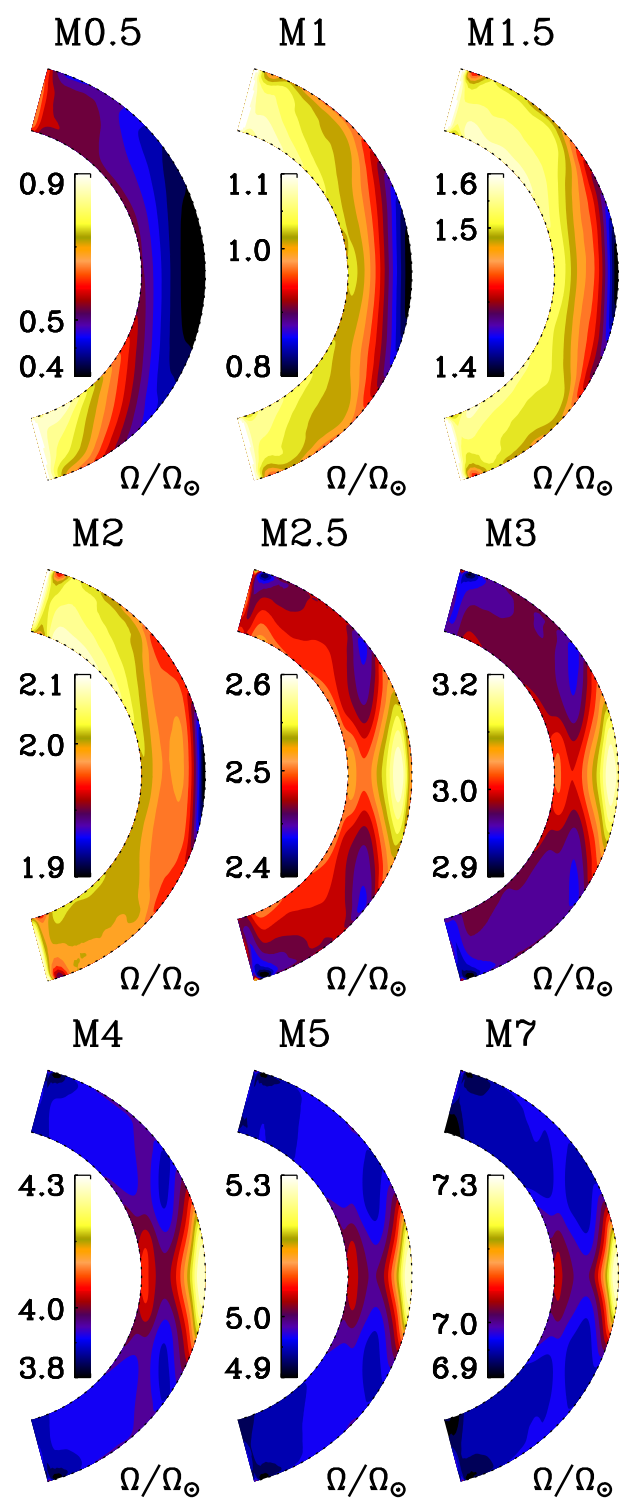

M5

$\mathrm{M} 7$
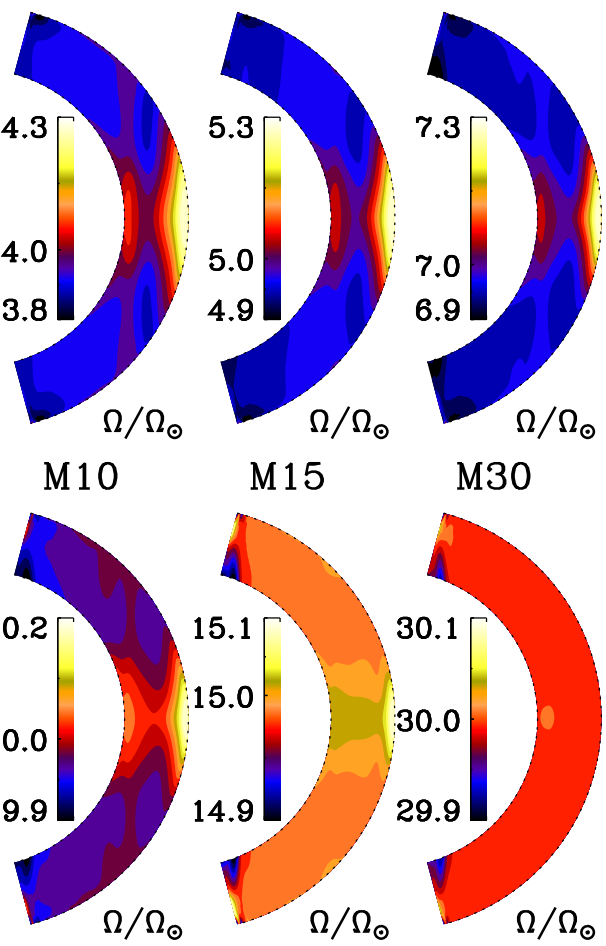

Fig. A.2. Normalized local rotation profile $\Omega / \Omega_{\odot}$ with $\Omega=\Omega_{0}+$ $\bar{u} / r \sin \theta$ for all MHD runs (Set M). The values of $\Omega$ have been calculated as a time average over the saturated state.

lyzed in the main part of the paper. In Figs. A.3 and A.4, we additionally show the diagonal components of the $\alpha$ tensor and $\beta$ tensor, respectively, in cylindrical coordinates to aid comparison with theoretical studies. 
J. Warnecke and M. J. Käpylä: Rotational dependence of turbulent transport coefficients

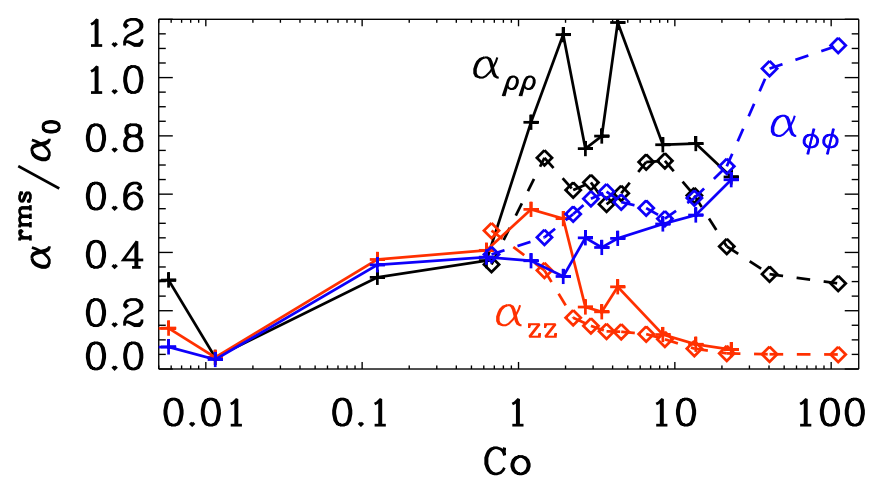

Fig. A.3. Same plot as the bottom panel of Fig. 6 but for the diagonal components of $\boldsymbol{\alpha}$ in cylindrical coordinates $(\rho, \phi, z)$ with $\alpha_{\rho \rho}$ (black lines), $\alpha_{z z}$ (red) and $\alpha_{\phi \phi}$ (blue).

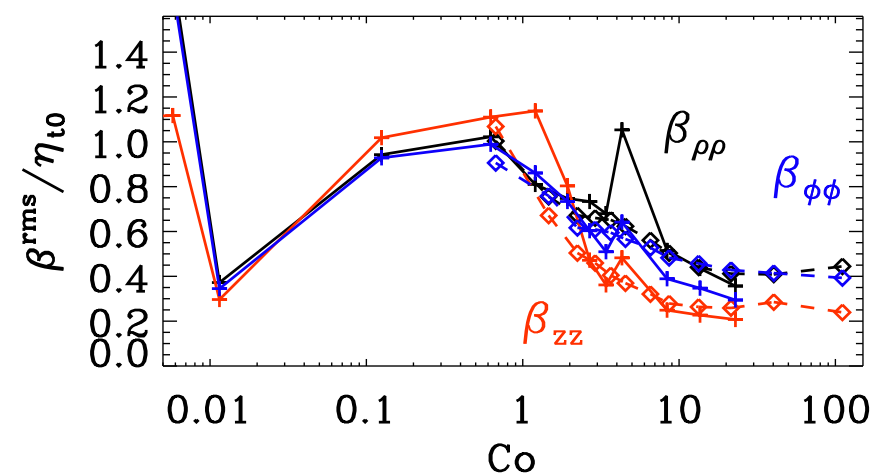

Fig. A.4. Same plot as the bottom panel of Fig. 10 but for the diagonal components of $\boldsymbol{\beta}$ in cylindrical coordinates $(\rho, \phi, z)$ with $\beta_{\rho \rho}$ (black lines), $\beta_{z z}$ (red) and $\beta_{\phi \phi}$ (blue).
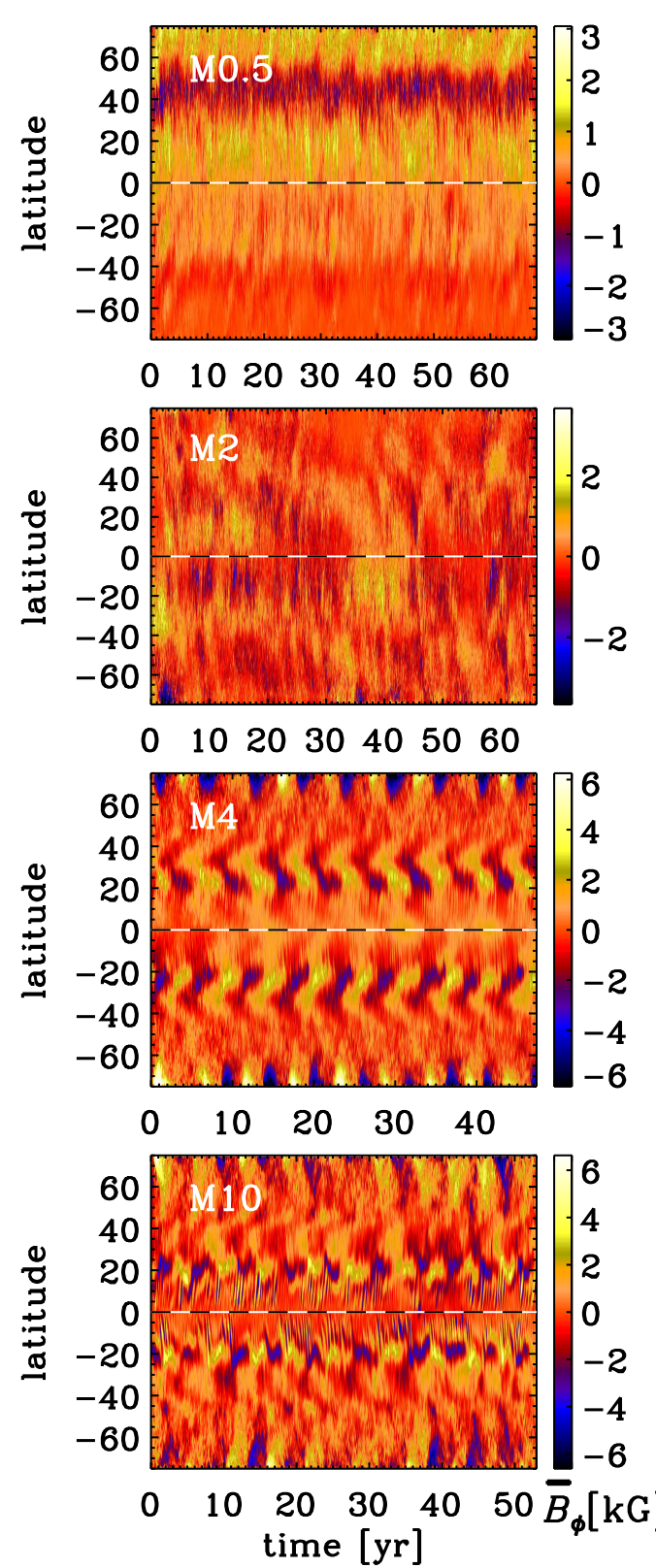
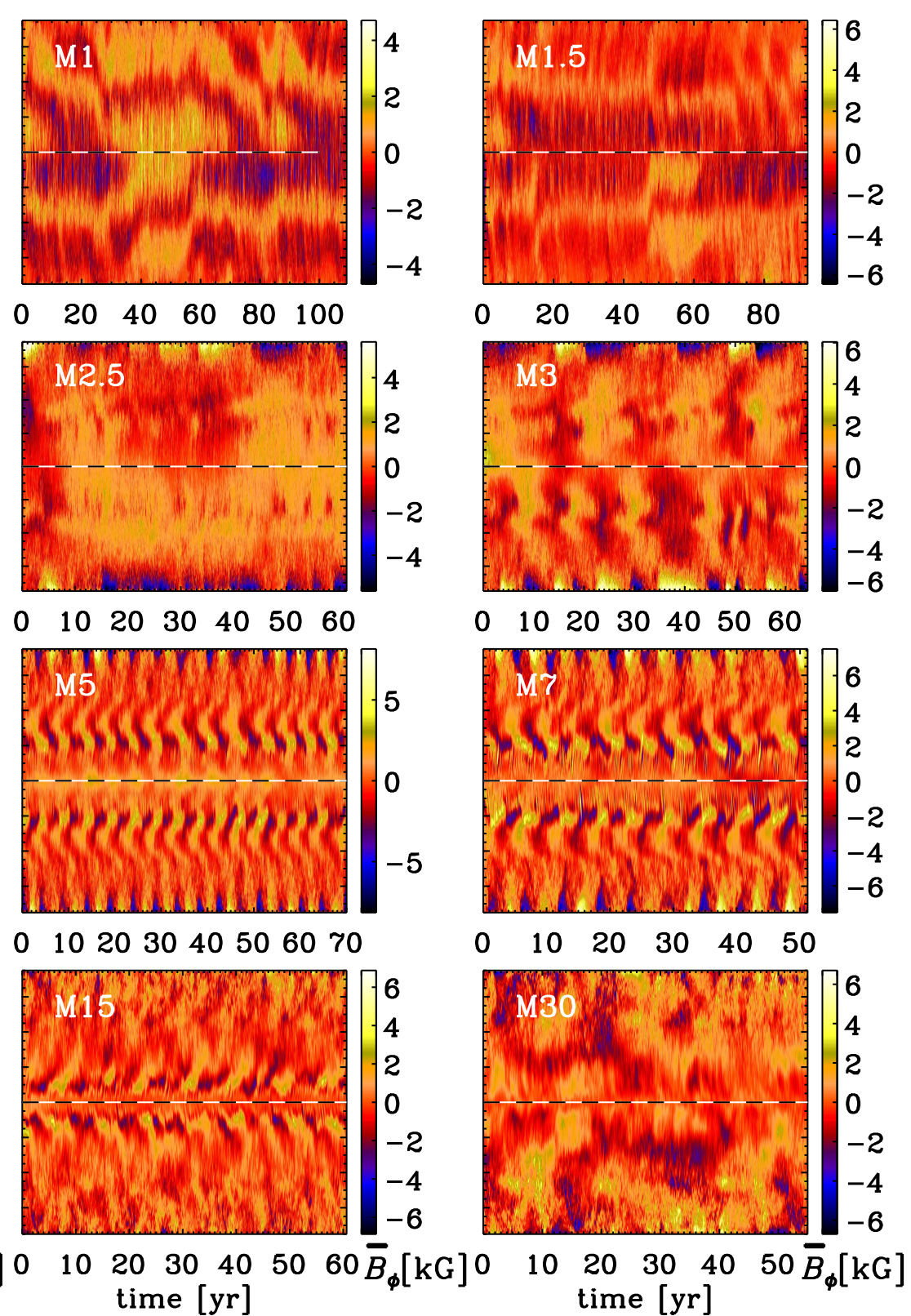

Fig. A.5. Mean azimuthal magnetic field $\bar{B}_{\phi}$ as a function of time in years and latitude near the surface $(r=0.98 R)$ for all MHD runs (Set M). The time interval shows the full duration of the saturated state for all runs. The black and white dashed horizontal line indicates the equator. 